Article

\title{
Design, Synthesis and Bioactivities of Novel Isoxazole-Containing Pyrazole Oxime Derivatives
}

\author{
Hong Dai ${ }^{1} \mathbb{B}^{\mathbb{D}}$, Wei Yao ${ }^{1}$, Yuan Fang ${ }^{2}$, Siyu Sun ${ }^{1}$, Yujun Shi ${ }^{1,2, *}$, Jia Chen ${ }^{1}$, Guoqing Jiang ${ }^{1, *}$ \\ and Jian $\mathrm{Shi}^{3, *}$ \\ 1 College of Chemistry and Chemical Engineering, Nantong University, Nantong 226019, China; \\ daihong_2015@aliyun.com (H.D.); yaowei265688@126.com (W.Y.); sunsiyu95@163.com (S.S.); \\ 15642891665@163.com (J.C.) \\ 2 School of Environmental and Chemical Engineering, Jiangsu University of Science and Technology, \\ Zhenjiang 212003, China; fyuan6586@aliyun.com \\ 3 Analysis and Testing Center, Nantong University, Nantong 226019, China \\ * Correspondence: syj@ntu.edu.cn (Y.S.); jguoj2016@163.com (G.J.); shi.j1@ntu.edu.cn (J.S.); \\ Tel./Fax: +86-513-8501-2851 (Y.S.); +86-513-8501-2851 (G.J.); +86-513-8501-2965 (J.S.)
}

Received: 9 November 2017; Accepted: 24 November 2017; Published: 27 November 2017

\begin{abstract}
In this study, in order to find novel biologically active pyrazole oxime derivatives, twenty-eight new pyrazole oxime compounds containing a substituted isoxazole ring were synthesized and evaluated for their acaricidaland insecticidal activities. Bioassays exhibited that some target compounds indicated good acaricidal and insecticidal activities against Tetranychus cinnabarinus, Aphis medicaginis, Mythimna separata, and Nilaparvata lugens. Especially, compounds $\mathbf{9 c}, \mathbf{9 h}, \mathbf{9 u}$, and 9v showed $100.00 \%, 90.56 \%, 90.78 \%$, and $90.62 \%$ insecticidal activities against $A$. medicaginis at the concentration of $20 \mu \mathrm{g} / \mathrm{mL}$, respectively, compounds $9 \mathbf{k}$ and $9 \mathbf{u}$ had $70.86 \%$ and $100.00 \%$ insecticidal activities against $M$. separata at $20 \mu \mathrm{g} / \mathrm{mL}$, respectively.
\end{abstract}

Keywords: isoxazole; pyrazole oxime; synthesis; bioactivity

\section{Introduction}

In the past few decades, heterocycles play a significant role in the research of agricultural and medicinal chemistry. The isoxazole skeleton, a crucial type of nitrogen-containing heterocycle, has been used in pesticide and drug design because of their various biological activities, such as insecticidal [1-3], herbicidal [4-6], fungicidal [7], antiviral [8-10], and anticancer activities [11]. Recently, Yu et al. obtained a series of 3,4,5-trisubstituted isoxazoles that were showing good insecticidal activities [12]. More recently, Sun et al. reported several series of isoxazole compounds carrying benzoylurea moiety displaying perfect insecticidal activities [13]. The widespread use of isoxazole-based compounds as a scaffold in the field of agriculture and medicine research endows the isoxazole ring as an important structural class.

On the other hand, pyrazole oxime derivatives are one of the hotspots in the design of new drugs due to their broad spectrum of insecticidal [14,15], acaricidal [16], fungicidal [17], anti-TMV [18], and antitumor activities [19]. In particular, the insecticidal and acaricidal activities have been widely investigated for their potential applications in agricultural production. For instance, Fenpyroximate (Figure 1), an excellent acaricide containing a pyrazole oxime moiety, is used to control some phytophagous mites, such as Tetranychus urticae Koch and Polyphagotarsonemus latus Banks [20,21]. Recently, Dai et al. reported that some pyrazole oximes possessed interesting insecticidal and acaricidal activity through modification of the esterified group of Fenpyroximate with thiazolylmethoxy or thiadiazolylmethoxy unit [22,23]. Wang and co-workers synthesized and evaluated the insecticidal activity of a series of pyrazole oxime ethers containing oxazole ring and found that some compounds 
showed good insecticidal properties against Aphis craccivora and Nilaparvata lugens [24]. Very recently, Dai and co-workers also obtained some pyrazole oximes owning wonderful acaricidal and insecticidal activities against Tetranychus cinnabarinus, Aphis medicaginis and Nilaparvata lugens by substituting the esterified aryl group of Fenpyroximate with oxadiazole ring [25]. This gave a great impetus to the search for biologically active molecules carrying the pyrazole oxime subunit.

Encouraged by the aforementioned facts, we envisioned that the introduction of a substituted isoxazole pharmacophore into the parent pyrazole oxime scaffold might produce some new compounds with multiple biological activities. In this study, we describe the design and synthesis of a number of novel pyrazole oxime derivatives bearing isoxazole ring (Figure 1). Moreover, all of the new compounds were tested for their acaricidal and insecticidal activities.

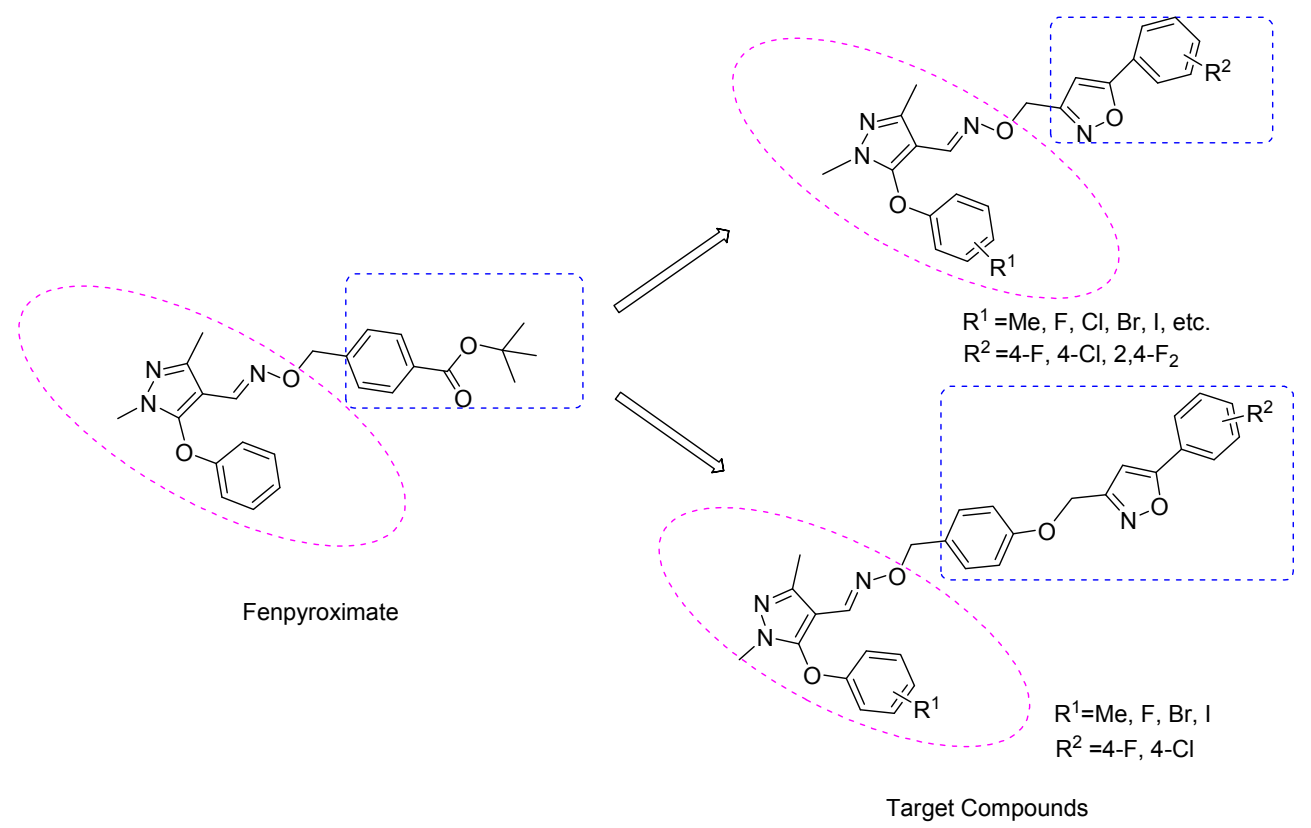

Figure 1. The design of the target molecules.

\section{Results and Discussion}

\subsection{Chemistry}

The general synthetic route of the title compounds $\mathbf{9 a - 9 v}$ was depicted in Scheme 1 . The key intermediates 3 were conveniently synthesized by two steps from 1,3-dimethyl-5-chloro- $1 H$ pyrazole-4-carbaldehyde (1). The condensation of compound $\mathbf{1}$ with various substituted phenols under basic conditions gave compounds $\mathbf{2}$, which were easily transformed into intermediates $\mathbf{3}$ by treatment with hydroxylamine hydrochloride using potassium hydroxide as the base. Intermediate 3-chloromethyl-5-substituted phenyl isoxazole (8) was prepared from compound 4. Compound 4 was easily reacted with dimethyl oxalate under basic conditions to obtain compound 5. Further reaction with hydroxylamine hydrochloride afforded compound 6 successfully. Next, a reaction with $\mathrm{LiAlH}_{4}$ was undertaken to form compound 7 in good yields. Further chlorination of compound 7 provided intermediate $\mathbf{8}$ smoothly by the addition of some drops of $N, N$-dimethylformamide. Finally, pyrazole oximes 3 were admixed with 3-chloromethyl-5-substituted phenyl isoxazole (8) in acetonitrile using potassium carbonate as alkali and cesium carbonate as catalyst to afford corresponding compounds $\mathbf{9 a - 9 v}$ in satisfactory yields (Scheme 1). The synthetic route for the target compounds 13a-13f is shown in Scheme 2. The important intermediates $\mathbf{1 2}$ were smoothly prepared in three steps from compound $\mathbf{8}$. The condensation of compound $\mathbf{8}$ with 4 -hydroxybenzaldehyde under basic condition afforded compounds 10, which were transformed to intermediates $\mathbf{1 1}$ by treatment with $\mathrm{LiAlH}_{4}$. Then, 
compounds 11 reacted with $\mathrm{SOCl}_{2}$ to give intermediates $\mathbf{1 2}$ conveniently. At last, intermediates 12 were treated with compound $\mathbf{8}$ in acetonitrile using potassium carbonate as alkali to obtain corresponding pyrazole oximes derivatives 13a-13f in good yields (Scheme 2). The target compounds $\mathbf{9 a - 9 v}$ and 13a-13f were effectively characterized by ${ }^{1} \mathrm{H}-\mathrm{NMR},{ }^{13} \mathrm{C}-\mathrm{NMR}$, and elemental analyses.
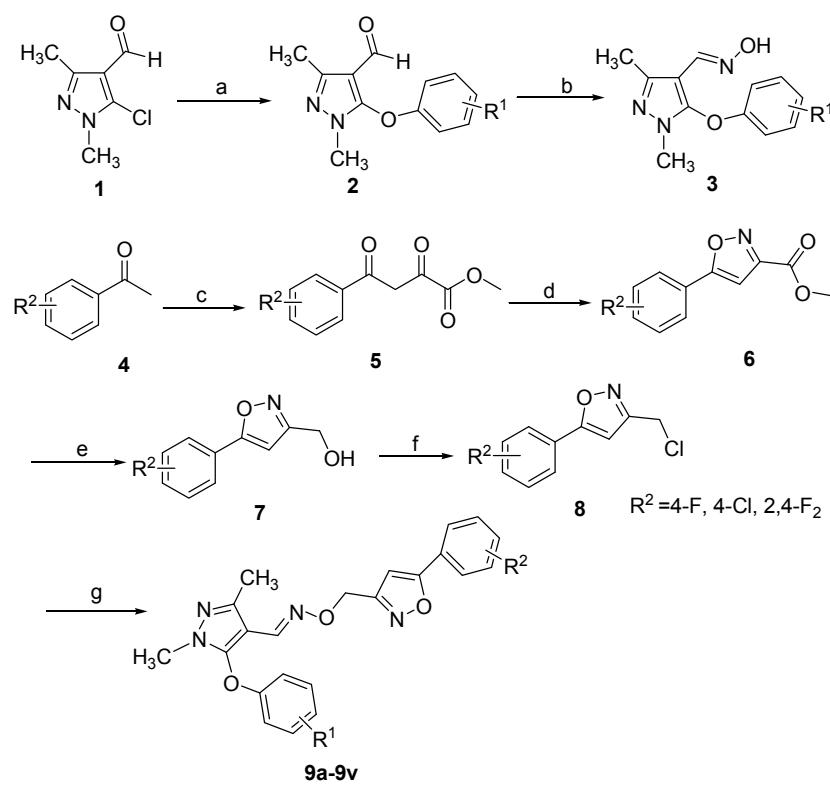

$\begin{array}{llll}\text { 9a: } R^{1}=2-M e, R^{2}=4-F & 9 g: R^{1}=4-B r, R^{2}=4-F & 9 m: R^{1}=2-F, R^{2}=4-C l & 9 r: R^{1}=4-O M e, R^{2}=2,4-F_{2} \\ \text { 9b: } R^{1}=4-M e, R^{2}=4-F & 9 h: R^{1}=4-I, R^{2}=4-F & 9 n: R^{1}=4-F, R^{2}=4-C l & 9 s: R^{1}=4-F, R^{2}=2,4-F_{2} \\ \text { 9c: } R^{1}=4-O M e, R^{2}=4-F & 9 i: R^{1}=2,4-F_{2}, R^{2}=4-F & 9 o: R^{1}=3-B r, R^{2}=4-C l & 9 t: R^{1}=4-C l, R^{2}=2,4-F_{2} \\ \text { 9d } R^{1}=2-C l, R^{2}=4-F & 9 j: R^{1}=2,4-C C_{2}, R^{2}=4-F & 9 p: R^{1}=4-I, R^{2}=4-C l & 9 u: R^{1}=4-B r, R^{2}=2,4-F_{2} \\ \text { 9e: } R^{1}=4-F, R^{2}=4-F & 9 k: R^{1}=4-O M e, R^{2}=4-C l & 9 q: R^{1}=2,4-F_{2}, R^{2}=4-C l & 9 v: R^{1}=4-I, R^{2}=2,4-F_{2} \\ \text { 9f: } R^{1}=4-C l, R^{2}=4-F & \text { 9I: } R^{1}=4-O C F_{3}, R^{2}=4-C l & 9 q: R^{1}\end{array}$

Scheme 1. Synthesis of the title compounds $9 a-9 v$. Reagents and conditions: (a) substituted phenol, $\mathrm{KOH}, \mathrm{DMF}$ or DMSO, $40{ }^{\circ} \mathrm{C}, 2-4 \mathrm{~h}, 105{ }^{\circ} \mathrm{C}, 8-24 \mathrm{~h}, 52-76 \%$ for 2 ; (b) $\mathrm{NH}_{2} \mathrm{OH} \cdot \mathrm{HCl}, \mathrm{KOH}, \mathrm{CH}_{3} \mathrm{OH}$, reflux, 7-22 h, 61-73\% for 3; (c) dimethyl oxalate, $\mathrm{CH}_{3} \mathrm{ONa}, \mathrm{CH}_{3} \mathrm{OH}, 60{ }^{\circ} \mathrm{C}, 6-8 \mathrm{~h}, 53-65 \%$ for 5; (d) $\mathrm{NH}_{2} \mathrm{OH} \cdot \mathrm{HCl}, \mathrm{CH}_{3} \mathrm{OH}, 60{ }^{\circ} \mathrm{C}, 7-10 \mathrm{~h}, 55-60 \%$ for 6; (e) $\mathrm{LiAlH}_{4}, \mathrm{THF}, 0{ }^{\circ} \mathrm{C}, 3-6 \mathrm{~h}, 70-76 \%$ for 7; (f) $\mathrm{SOCl}_{2}, \mathrm{DMF}, \mathrm{CH}_{2} \mathrm{Cl}_{2}, 0{ }^{\circ} \mathrm{C}, 4-7 \mathrm{~h}, 73-80 \%$ for 8; (g) compound 3, $\mathrm{K}_{2} \mathrm{CO}_{3}, \mathrm{Cs}_{2} \mathrm{CO}_{3}, \mathrm{CH}_{3} \mathrm{CN}$, reflux, 8-19 h, 47-63\% for 9.

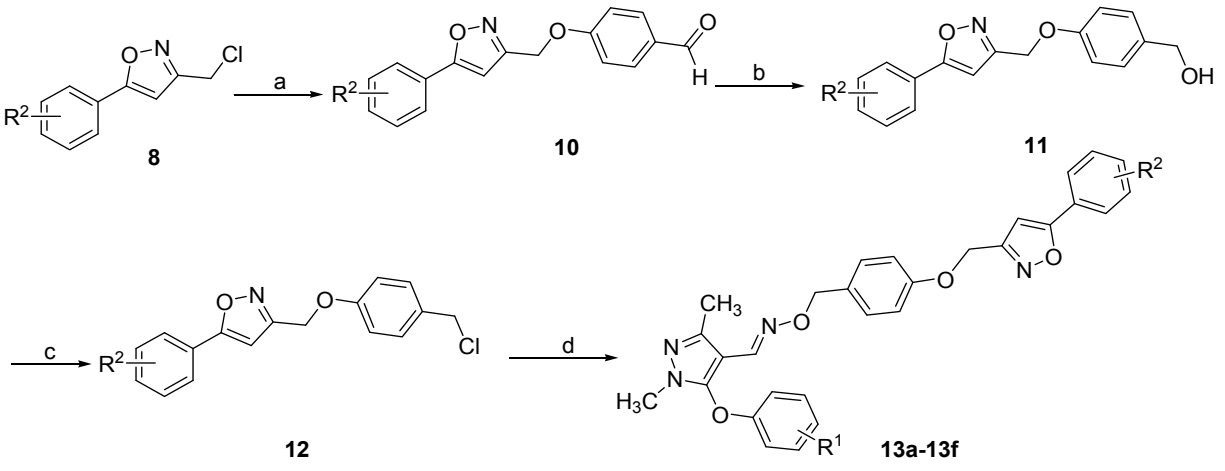

$$
\begin{array}{ll}
\text { 13a: } R^{1}=4-M e, R^{2}=4-F & 13 d R^{1}=4-I, R^{2}=4-F \\
\text { 13b: } R^{1}=4-F, R^{2}=4-F & 13 e: R^{1}=4-B r, R^{2}=4-C l \\
\text { 13c: } R^{1}=4-B r, R^{2}=4-F & 13 f: R^{1}=4-I, R^{2}=4-C l
\end{array}
$$

Scheme 2. Synthesis of the title compounds 13a-13f. Reagents and conditions: (a) 4-hydroxybenzaldehyde, $\mathrm{Cs}_{2} \mathrm{CO}_{3}, \mathrm{CH}_{3} \mathrm{CN}$, reflux, $5 \mathrm{~h}, 71-77 \%$ for 10; (b) $\mathrm{LiAlH}_{4}, \mathrm{THF}, 0{ }^{\circ} \mathrm{C}, 30 \mathrm{~min}, 68-72 \%$ for 11; (c) $\mathrm{SOCl}_{2}$, DMF, $\mathrm{CH}_{2} \mathrm{Cl}_{2}, 0{ }^{\circ} \mathrm{C}, 6-8 \mathrm{~h}, 62-65 \%$ for 12; and, (d) compound $3, \mathrm{~K}_{2} \mathrm{CO}_{3}, \mathrm{CH}_{3} \mathrm{CN}$, reflux, 10-15 h, $46-56 \%$ for 13 . 


\subsection{Biological Activities}

The synthesized compounds $\mathbf{9 a}-\mathbf{9 v}$ and $\mathbf{1 3 a} \mathbf{a}-\mathbf{1 3 f}$ were evaluated for insecticidal activities against Aphis medicaginis, Mythimna separata and Nilaparvata lugens, and acaricidal activity against Tetranychus cinnabarinus using known procedures, and Chlorantraniliprole, Pyridalyl, Abamectin and Fenpyroximate were used as the positive controls, respectively. As indicated in Table 1, some title compounds showed moderate to good acaricidal activity against $T$. cinnabarinus at a concentration of $500 \mu \mathrm{g} / \mathrm{mL}$. The mortalities of compounds $\mathbf{9 e}$ and $\mathbf{9 q}$ were $80.46 \%$ and $50.38 \%$, respectively. Besides acaricidal potencies, most title compounds displayed wonderful insecticidal activities against A. medicaginis at a dosgae of $500 \mu \mathrm{g} / \mathrm{mL}$, for example, compounds $\mathbf{9 b}, \mathbf{9 c}, \mathbf{9 d}, \mathbf{9 e}, \mathbf{9 g}, \mathbf{9 h}, \mathbf{9 i}, \mathbf{9 j}$, 9k, 9q, 9r, 9s, 9t, 9u, and $9 v$ all possessed $100.00 \%$ inhibition rates, respectively, which were equal to that of the control Chlorantraniliprole. Furthermore, some aimed compounds exhibited potent insecticidal activities against $A$. medicaginis when the dosage was lowered to $100 \mu \mathrm{g} / \mathrm{mL}$. For instance, compounds $\mathbf{9 b}, \mathbf{9 c}, \mathbf{9 d}, \mathbf{9 g}, \mathbf{9 h}, \mathbf{9 i}, \mathbf{9 j}, \mathbf{9 u}$, and $\mathbf{9 v}$ all owned $100.00 \%$ insecticidal activity against A. medicaginis, respectively, which were comparable to that of the control Chlorantraniliprole. Even when the dosage was reduced to $20 \mu \mathrm{g} / \mathrm{mL}$, some compounds still had satisfactory insecticidal activity against $A$. medicaginis, and the mortalities of compounds $9 \mathbf{c}, 9 \mathbf{h}, \mathbf{9 u}$, and $9 \mathbf{v}$ were $100.00 \%, 90.56 \%$, $90.78 \%$, and $90.62 \%$, respectively. From the above insecticidal activity data, we can find that the substituents $\left(R^{1}\right)$ on the phenyl ring may have an impact on the activities. When $R^{2}$ is 4 -fluoro or 2,4-difluoro, the substituent $\left(R^{1}\right)$ at 4-position of phenyl ring was methoxy $(9 \mathrm{c})$, halogen $(9 \mathrm{~g}, 9 \mathbf{h}, \mathbf{9 u}$, and $\mathbf{9 v}$ ), or 2,4-position of phenyl ring was fluoro or chloro (9i and $\mathbf{9 j})$, it was more favorable to the insecticidal activity against $A$. medicaginis at the dosage of $20 \mu \mathrm{g} / \mathrm{mL}$. Table 2 demonstrated that most target compounds showed excellent larvicidal activity against $M$. separata at a concentration of $500 \mu \mathrm{g} / \mathrm{mL}$. Moreover, some of them had moderate to good larvicidal activity against $M$. separata when the concentration came to $100 \mu \mathrm{g} / \mathrm{mL}$, among these derivatives, compounds $\mathbf{9 c}$, $9 \mathbf{k}$, and $\mathbf{9 u}$ all exhibited $100.00 \%$ inhibition rates, respectively, which were comparable to that of the control Pyridalyl. When the concentration arrived at $20 \mu \mathrm{g} / \mathrm{mL}$, compounds $\mathbf{9 k}$ and $\mathbf{9 u}$ still indicated potential inhibitory activities against $M$. separata, with the inhibition rates being $70.86 \%$ and $100.00 \%$, respectively. When $\mathrm{R}^{2}$ is 4-fluoro or 2,4-difluoro, the substituent $\left(R^{1}\right)$ at 4-position of phenyl ring was methoxy (9k) or bromo $(9 \mathbf{u})$, it was more favorable to the insecticidal activity against $M$. separata at $20 \mu \mathrm{g} / \mathrm{mL}$. Interestingly, some designed compounds possessed wonderful inhibitory activities against $N$. lugens besides good insecticidal activities against $A$. medicaginis and M. separata. Among them, compounds 9c, 9d, 9i, 9k, 9r, 9s, and $9 \mathbf{t}$ all had $100.00 \%$ inhibition rates against $N$. lugens at $500 \mu \mathrm{g} / \mathrm{mL}$. When the dosage was reduced to $100 \mu \mathrm{g} / \mathrm{mL}$, some title compounds were still active against $N$. lugens, especially, the inhibition rates of compounds $9 \mathbf{e}$ and $\mathbf{9 g}$ were $75.23 \%$ and $75.76 \%$. From the data shown in Tables 1 and 2, we found that the structure-insecticidal activity relationship of some obtained compounds against $N$. lugens is similar to structure-insecticidal activity relationship of some compounds against A. medicaginis. When $\mathrm{R}^{2}$ is 4 -fluoro, the substituent $\left(\mathrm{R}^{1}\right)$ was 4 -fluoro $(9 \mathrm{e})$ or 4 -bromo $(9 \mathrm{~g})$, it was more advantageous to increase the insecticidal activity against $N$. lugens at $100 \mu \mathrm{g} / \mathrm{mL}$ than other substituents. The data presented in Tables 1 and 2 also displayed that compounds $9 \mathbf{c}, \mathbf{9 g}, \mathbf{9 h}, \mathbf{9 i}$, 9j, 9k, 9r, 9s, and $9 \mathbf{t}$ exhibited exciting insecticidal effects against $N$. lugens and $M$. separata beyond wonderful insecticidal activities against $A$. medicaginis at the dosage of $500 \mu \mathrm{g} / \mathrm{mL}$. At the same time, compound $\mathbf{9 e}$ had good acaricidal activity against $T$. cinnabarinus besides potent insecticidal activities against A. medicaginis, M. separata, and N. lugens at $500 \mu \mathrm{g} / \mathrm{mL}$. From the data listed in Tables 1 and 2, we can find that some benzyloxy-linked isoxazole derivatives also possessed good insecticidal activities against $A$. medicaginis, $M$. separata, and $N$. lugens at the dosage of $500 \mu \mathrm{g} / \mathrm{mL}$. For example, compounds $\mathbf{1 3 c}$ and $\mathbf{1 3 f}$ both had $100.00 \%$ insecticidal activities against $A$. medicaginis, which were similar to that of the control Chlorantraniliprole. Compounds 13a, 13b, 13c, 13e, and 13f all displayed $100.00 \%$ insecticidal activities against $M$. separata, respectively, which were equal to that of the control Pyridalyl. In addition, compound $\mathbf{1 3 d}$ indicated $100.00 \%$ inhibitory activity against $N$. lugens, which was near to that of the control Abamectin. All of the above data implied that the bioactivity spectrum 
of pyrazole oxime derivatives was significantly improved by introducing the important isoxazole ring. This research indicated that these target compounds may function as potential lead structures for the discovery of novel pesticides in future.

Table 1. Acaricidal and insecticidal activities of target compounds $\mathbf{9 a}-\mathbf{9 v}$ and $\mathbf{1 3 a} \mathbf{a}-\mathbf{1 3 f}$ (mortality, \%).

\begin{tabular}{|c|c|c|c|c|c|}
\hline \multirow{2}{*}{ Compd. } & \multicolumn{2}{|c|}{ Tetranychus cinnabarinus } & \multicolumn{3}{|c|}{ Aphis medicaginis } \\
\hline & $500 \mu \mathrm{g} / \mathrm{mL}$ & $100 \mu \mathrm{g} / \mathrm{mL}$ & $500 \mu \mathrm{g} / \mathrm{mL}$ & $100 \mu \mathrm{g} / \mathrm{mL}$ & $20 \mu \mathrm{g} / \mathrm{mL}$ \\
\hline 9a & 0 & $-b$ & $90.52 \pm 0.72$ & $40.54 \pm 1.22$ & 0 \\
\hline $9 b$ & 0 & - & $100.00 \pm 0.00$ & $100.00 \pm 0.00$ & $40.89 \pm 0.57$ \\
\hline $9 \mathrm{c}$ & 0 & - & $100.00 \pm 0.00$ & $100.00 \pm 0.00$ & $100.00 \pm 0.00$ \\
\hline $9 d$ & 0 & - & $100.00 \pm 0.00$ & $100.00 \pm 0.00$ & $60.36 \pm 1.08$ \\
\hline $9 e$ & $80.46 \pm 0.65^{\mathrm{a}}$ & $50.49 \pm 1.78$ & $100.00 \pm 0.00$ & $90.66 \pm 0.53$ & $50.67 \pm 1.36$ \\
\hline $9 f$ & 0 & - & $50.33 \pm 1.59$ & 0 & - \\
\hline $9 g$ & 0 & - & $100.00 \pm 0.00$ & $100.00 \pm 0.00$ & $80.73 \pm 0.71$ \\
\hline $9 \mathrm{~h}$ & 0 & - & $100.00 \pm 0.00$ & $100.00 \pm 0.00$ & $90.56 \pm 0.82$ \\
\hline $9 \mathrm{i}$ & 0 & - & $100.00 \pm 0.00$ & $100.00 \pm 0.00$ & $85.83 \pm 0.69$ \\
\hline $9 j$ & 0 & - & $100.00 \pm 0.00$ & $100.00 \pm 0.00$ & $80.28 \pm 1.21$ \\
\hline $9 k$ & 0 & - & $100.00 \pm 0.00$ & 0 & - \\
\hline 91 & 0 & - & 0 & - & - \\
\hline $9 m$ & 0 & - & $50.72 \pm 1.37$ & 0 & - \\
\hline $9 n$ & 0 & - & 0 & - & - \\
\hline 90 & 0 & - & 0 & - & - \\
\hline $9 p$ & 0 & - & 0 & - & - \\
\hline $9 q$ & $50.38 \pm 1.23$ & 0 & $100.00 \pm 0.00$ & 0 & - \\
\hline $9 \mathrm{r}$ & 0 & - & $100.00 \pm 0.00$ & $70.89 \pm 1.25$ & 0 \\
\hline $9 \mathrm{~s}$ & 0 & - & $100.00 \pm 0.00$ & $40.57 \pm 0.68$ & - \\
\hline $9 t$ & 0 & - & $100.00 \pm 0.00$ & 0 & - \\
\hline $9 \mathbf{u}$ & 0 & - & $100.00 \pm 0.00$ & $100.00 \pm 0.00$ & $90.78 \pm 1.35$ \\
\hline $9 v$ & 0 & - & $100.00 \pm 0.00$ & $100.00 \pm 0.00$ & $90.62 \pm 0.96$ \\
\hline $13 a$ & 0 & - & 0 & - & - \\
\hline $13 b$ & 0 & - & 0 & - & - \\
\hline $13 \mathrm{c}$ & $20.26 \pm 1.61$ & - & $100.00 \pm 0.00$ & $20.92 \pm 0.52$ & - \\
\hline $13 d$ & 0 & - & 0 & - & - \\
\hline $13 e$ & 0 & - & 0 & - & - \\
\hline $13 f$ & 0 & - & $100.00 \pm 0.00$ & $30.81 \pm 1.47$ & - \\
\hline Fenpyroximate & $100.00 \pm 0.00$ & $100.00 \pm 0.00$ & - & - & - \\
\hline Chlorantraniliprole & - & - & $100.00 \pm 0.00$ & $100.00 \pm 0.00$ & $100.00 \pm 0.00$ \\
\hline
\end{tabular}

a Each value represents the mean \pm standard error of three replications. ${ }^{\mathrm{b}}$ " - $"$ " refers to "not tested".

Table 2. Insecticidal activities of title compounds $\mathbf{9 a}-\mathbf{9 v}$ and 13a-13f (mortality, \%).

\begin{tabular}{|c|c|c|c|c|c|c|}
\hline \multirow{2}{*}{ Compd. } & \multicolumn{3}{|c|}{ Mythimna separata } & \multicolumn{3}{|c|}{ Nilaparvata lugens } \\
\hline & $500 \mu \mathrm{g} / \mathrm{mL}$ & $100 \mu \mathrm{g} / \mathrm{mL}$ & $20 \mu \mathrm{g} / \mathrm{mL}$ & $500 \mu \mathrm{g} / \mathrm{mL}$ & $100 \mu \mathrm{g} / \mathrm{mL}$ & $20 \mu \mathrm{g} / \mathrm{mL}$ \\
\hline $9 a$ & 0 & $-\mathrm{b}$ & - & $85.54 \pm 1.32$ & 0 & - \\
\hline $9 b$ & $90.37 \pm 0.85^{a}$ & 0 & - & 0 & - & - \\
\hline $9 \mathrm{c}$ & $100.00 \pm 0.00$ & $100.00 \pm 0.00$ & $40.57 \pm 0.76$ & $100.00 \pm 0.00$ & 0 & - \\
\hline $9 d$ & $60.29 \pm 1.03$ & 0 & - & $100.00 \pm 0.00$ & 0 & - \\
\hline $9 e$ & $50.55 \pm 0.92$ & 0 & - & $90.26 \pm 1.45$ & $75.23 \pm 0.69$ & $30.87 \pm 1.43$ \\
\hline $9 f$ & $80.76 \pm 0.65$ & 0 & - & $50.32 \pm 1.21$ & 0 & - \\
\hline $9 g$ & $80.32 \pm 0.82$ & $50.43 \pm 0.73$ & 0 & $80.79 \pm 0.87$ & $75.76 \pm 0.75$ & 0 \\
\hline $9 h$ & $100.00 \pm 0.00$ & $40.56 \pm 0.47$ & - & $95.23 \pm 0.65$ & $50.43 \pm 1.58$ & 0 \\
\hline $9 \mathrm{i}$ & $100.00 \pm 0.00$ & $30.19 \pm 1.58$ & - & $100.00 \pm 0.00$ & 0 & - \\
\hline $9 j$ & $90.74 \pm 0.57$ & 0 & - & $90.86 \pm 0.71$ & 0 & - \\
\hline $9 k$ & $100.00 \pm 0.00$ & $100.00 \pm 0.00$ & $70.86 \pm 0.63$ & $100.00 \pm 0.00$ & 0 & - \\
\hline 91 & 0 & - & - & $50.43 \pm 0.54$ & 0 & - \\
\hline $9 m$ & 0 & - & - & $80.31 \pm 1.29$ & 0 & - \\
\hline $9 n$ & 0 & - & - & 0 & - & - \\
\hline
\end{tabular}


Table 2. Cont.

\begin{tabular}{|c|c|c|c|c|c|c|}
\hline \multirow{2}{*}{ Compd. } & \multicolumn{3}{|c|}{ Mythimna separata } & \multicolumn{3}{|c|}{ Nilaparvata lugens } \\
\hline & $500 \mu \mathrm{g} / \mathrm{mL}$ & $100 \mu \mathrm{g} / \mathrm{mL}$ & $20 \mu \mathrm{g} / \mathrm{mL}$ & $500 \mu \mathrm{g} / \mathrm{mL}$ & $100 \mu \mathrm{g} / \mathrm{mL}$ & $20 \mu \mathrm{g} / \mathrm{mL}$ \\
\hline 90 & $100.00 \pm 0.00$ & 0 & - & 0 & - & - \\
\hline $9 p$ & $100.00 \pm 0.00$ & $60.58 \pm 1.21$ & 0 & 0 & - & - \\
\hline $9 q$ & $100.00 \pm 0.00$ & 0 & - & $70.32 \pm 0.86$ & 0 & - \\
\hline $9 \mathrm{r}$ & $100.00 \pm 0.00$ & $30.48 \pm 0.65$ & - & $100.00 \pm 0.00$ & $40.87 \pm 0.73$ & - \\
\hline $9 \mathrm{~s}$ & $100.00 \pm 0.00$ & 0 & - & $100.00 \pm 0.00$ & 0 & - \\
\hline $9 t$ & $100.00 \pm 0.00$ & $70.18 \pm 1.43$ & $30.27 \pm 1.22$ & $100.00 \pm 0.00$ & $30.75 \pm 0.82$ & - \\
\hline $9 u$ & $100.00 \pm 0.00$ & $100.00 \pm 0.00$ & $100.00 \pm 0.00$ & 0 & - & - \\
\hline $9 v$ & $100.00 \pm 0.00$ & $50.21 \pm 1.56$ & 0 & 0 & - & - \\
\hline $13 a$ & $100.00 \pm 0.00$ & $40.43 \pm 1.81$ & - & 0 & - & - \\
\hline $13 b$ & $100.00 \pm 0.00$ & $30.61 \pm 0.63$ & - & 0 & - & - \\
\hline $13 c$ & $100.00 \pm 0.00$ & 0 & - & $20.68 \pm 1.21$ & - & - \\
\hline $13 d$ & $70.91 \pm 0.89$ & 0 & - & $100.00 \pm 0.00$ & $20.65 \pm 0.53$ & - \\
\hline $13 e$ & $100.00 \pm 0.00$ & 0 & - & $30.21 \pm 1.12$ & - & - \\
\hline $13 f$ & $100.00 \pm 0.00$ & 0 & - & $20.57 \pm 0.43$ & - & - \\
\hline Pyridalyl & $100.00 \pm 0.00$ & $100.00 \pm 0.00$ & $100.00 \pm 0.00$ & - & - & - \\
\hline Abamectin & - & - & - & $100.00 \pm 0.00$ & $100.00 \pm 0.00$ & $100.00 \pm 0.00$ \\
\hline
\end{tabular}

a Each value represents the mean \pm standard error of three replications. ${ }^{b}$ " - " refers to "not tested".

\section{Experimental Section}

\subsection{Chemistry}

\subsubsection{General Procedures}

All of the reagents were chemically pure and solvents were dried according to standard methods. ${ }^{1} \mathrm{H}-\mathrm{NMR}$ and ${ }^{13} \mathrm{C}-\mathrm{NMR}$ spectra were obtained on a Bruker AV400 spectrometer $\left(400 \mathrm{MHz},{ }^{1} \mathrm{H}\right.$; $100 \mathrm{MHz},{ }^{13} \mathrm{C}$, Bruker, Billerica, MA, USA) in $\mathrm{CDCl}_{3}$ with tetramethylsilane as the internal standard. The melting points were determined on an X-4 binocular microscope melting point apparatus (Beijing Tech Instrument Co., Beijing, China) and are uncorrected. Elemental analyses were determined on a Yanaco CHN Corder MT-3 elmental analyzer (Yanaco, Kyoto, Japan). The reactions were monitored by analytical thin-layer chromatography (TLC) with ultraviolet (UV) light and TLC was carried out on silica gel $\mathrm{GF}_{254}$. The intermediate 5-chloropyrazole aldehyde $\mathbf{1}$ was synthesized according to the reported procedure [26]. The intermediates $\mathbf{5}$ and $\mathbf{6}$ were prepared by the literature method [27].

\subsubsection{General Procedure for the Preparation of 2}

To a well stirred solution of substituted phenol (30 mmol) in DMF or DMSO (30 mL), KOH was added ( $40 \mathrm{mmol}$ ) at room temperature. The resulting mixture was heated to $40^{\circ} \mathrm{C}$ for $2-4 \mathrm{~h}$, and then compound $1(20 \mathrm{mmol})$ was added thereto. The reaction solution was heated to $105^{\circ} \mathrm{C}$ for $8-24 \mathrm{~h}$. After being cooled to room temperature, the mixture was poured into water and extracted with ethyl acetate $(3 \times 50 \mathrm{~mL})$. The combined extracts were dried over anhydrous magnesium sulfate, filtered, and evaporated to afford intermediate 2 , with yields ranging from $52 \%$ to $76 \%$ [25].

\subsubsection{General Procedure for the Preparation of 3}

To a solution of hydroxylamine hydrochloride $(30 \mathrm{mmol})$ in anhydrous methanol $(60 \mathrm{~mL})$ at room temperature, was added $\mathrm{KOH}(40 \mathrm{mmol})$ in portions, and the mixture was stirred at room temperature for $20 \mathrm{~min}$. To the above solution was added intermediate $\mathbf{2}$, the reaction mixture was heated to reflux for 7-22 $\mathrm{h}$. After being cooled to room temperature, the mixture was poured into water (100 $\mathrm{mL})$, and the solid precipitate was filtered, washed with water, and dried to give corresponding 5 -substituted phenoxy pyrazole oximes 3 , with yields ranging from $61 \%$ to $73 \%$ [25]. 


\subsubsection{General Procedure for the Preparation of 7}

To a well stirred cold $\left(0{ }^{\circ} \mathrm{C}\right)$ solution of intermediate $6(4 \mathrm{mmol})$ and THF $(60 \mathrm{~mL})$, was added $\mathrm{LiAlH}_{4}(10 \mathrm{mmol})$ in three portions and the reaction mixture was stirred at $0{ }^{\circ} \mathrm{C}$ for $3-6 \mathrm{~h}$. To the above solution, was added ice water $(40 \mathrm{~mL})$. After the solid precipitate was filtered, the filtrate was extracted with ethyl acetate $(3 \times 50 \mathrm{~mL})$. The combined extracts were dried over anhydrous sodium sulfate, filtered, and concentrated to produce compound 7, with yields ranging from $70 \%$ to $76 \%$ [28].

\subsubsection{General Procedure for the Preparation of 8}

To a well stirred cold $\left({ }^{\circ} \mathrm{C}\right)$ solution of compound $7(20 \mathrm{mmol})$ in $\mathrm{CH}_{2} \mathrm{Cl}_{2}(50 \mathrm{~mL})$, was added dropwise a mixture of thionyl chloride $(40 \mathrm{mmol})$ in $\mathrm{CH}_{2} \mathrm{Cl}_{2}(15 \mathrm{~mL})$. Then, several drops of DMF was added thereto. The resulting mixture was stirred at $0{ }^{\circ} \mathrm{C}$ for $4-7 \mathrm{~h}$. To the above solution, was added ice water $(50 \mathrm{~mL})$, and $\mathrm{pH}$ value of the mixture was adjusted to 6 by saturated sodium bicarbonate solution. The separated organic layer was dried over anhydrous sodium sulfate, filtered, and concentrated to afford the corresponding compound 8, with yields ranging from $73 \%$ to $80 \%$ [28].

\subsubsection{General Procedure for the Preparation of $\mathbf{9 a}-\mathbf{9 v}$}

To a mixture of intermediate $8(4 \mathrm{mmol})$, compound $3(5 \mathrm{mmol})$, and potassium carbonate (12 mmol) in acetonitrile $(30 \mathrm{~mL})$ at room temperature, was added cesium carbonate $(1 \mathrm{mmol})$. The resulting mixture was heated to reflux for 8-19 h. The reaction mixture was allowed to cool at room temperature and filtered. The solvent was evaporated under reduced pressure, and the residue was admixed with water $(50 \mathrm{~mL})$ and extracted with ethyl acetate $(3 \times 50 \mathrm{~mL})$. The combined organic layer was washed with water $(3 \times 20 \mathrm{~mL})$, dried over anhydrous sodium sulfate, filtered, and concentrated. The residue was purified by silica gel column chromatography using a mixture of petroleum ether and ethyl acetate as an eluent to produce the target compounds $\mathbf{9 a}-\mathbf{9 v}$, with yields ranging from $47 \%$ to $63 \%$. Pyrazole oxime derivatives $9 \mathbf{a}-9 \mathbf{v}$ were novel, and the physical and spectral data for these compounds are listed below. ${ }^{1} \mathrm{H}-\mathrm{NMR}$ and ${ }^{13} \mathrm{C}-\mathrm{NMR}$ spectra are provided in the Supplementary Materials.

Data for 9a. Yellow solid, yield 51\%, m.p.: 93-94 ${ }^{\circ} \mathrm{C} .{ }^{1} \mathrm{H}-\mathrm{NMR}\left(400 \mathrm{MHz}, \mathrm{DMSO}-d_{6}\right): \delta 7.87-7.91(\mathrm{~m}, 2 \mathrm{H}$, Ar-H), $7.75(\mathrm{~s}, 1 \mathrm{H}, \mathrm{CH}=\mathrm{N}), 6.98-7.40(\mathrm{~m}, 5 \mathrm{H}, \mathrm{Ar}-\mathrm{H}), 6.80(\mathrm{~s}, 1 \mathrm{H}$, Isoxazole-H), $6.56(\mathrm{~d}, J=8.0 \mathrm{~Hz}, 1 \mathrm{H}$, $\mathrm{Ar}-\mathrm{H}), 5.00\left(\mathrm{~s}, 2 \mathrm{H}, \mathrm{CH}_{2}\right), 3.55\left(\mathrm{~s}, 3 \mathrm{H}, \mathrm{N}-\mathrm{CH}_{3}\right), 2.32\left(\mathrm{~s}, 3 \mathrm{H}, \mathrm{CH}_{3}\right), 2.24\left(\mathrm{~s}, 3 \mathrm{H}, \mathrm{CH}_{3}\right) .{ }^{13} \mathrm{C}-\mathrm{NMR}(100 \mathrm{MHz}$, $\left.\mathrm{CDCl}_{3}\right): \delta 168.9,165.0,162.5,162.1,154.9,148.4,147.0,141.7,131.6,127.9,127.1,126.7,123.8,123.6,116.3$, 116.0, 113.3, 99.5, 98.9, 67.0, 34.1, 16.1, 14.7. Anal. Calcd for $\mathrm{C}_{23} \mathrm{H}_{21} \mathrm{FN}_{4} \mathrm{O}_{3}: \mathrm{C}, 65.70 ; \mathrm{H}, 5.03 ; \mathrm{N}, 13.33$. Found: $\mathrm{C}, 65.56 ; \mathrm{H}, 5.16 ; \mathrm{N}, 13.18$.

Data for 9 b. White solid, yield 53\%, m.p.: $96-97^{\circ} \mathrm{C} .{ }^{1} \mathrm{H}-\mathrm{NMR}\left(400 \mathrm{MHz}, \mathrm{CDCl}_{3}\right): \delta 7.83(\mathrm{~s}, 1 \mathrm{H}, \mathrm{CH}=\mathrm{N})$, $7.68(\mathrm{~d}, J=8.8 \mathrm{~Hz}, 2 \mathrm{H}, \mathrm{Ar}-\mathrm{H}), 7.43(\mathrm{~d}, J=8.4 \mathrm{~Hz}, 2 \mathrm{H}, \mathrm{Ar}-\mathrm{H}), 7.09$ (d, J = 8.0 Hz, 2H, Ar-H), 6.79 (d, $J=8.8 \mathrm{~Hz}, 2 \mathrm{H}, \mathrm{Ar}-\mathrm{H}), 6.47\left(\mathrm{~s}, 1 \mathrm{H}\right.$, Isoxazole-H), $5.10\left(\mathrm{~s}, 2 \mathrm{H}, \mathrm{CH}_{2}\right), 3.60\left(\mathrm{~s}, 3 \mathrm{H}, \mathrm{N}-\mathrm{CH}_{3}\right), 2.38(\mathrm{~s}, 3 \mathrm{H}$, $\left.\mathrm{CH}_{3}\right), 2.27\left(\mathrm{~s}, 3 \mathrm{H}, \mathrm{CH}_{3}\right) .{ }^{13} \mathrm{C}-\mathrm{NMR}\left(100 \mathrm{MHz}_{2} \mathrm{CDCl}_{3}\right): \delta 168.8,162.1,154.7,148.3,146.9,141.8,136.2$, 133.3, 130.6, 130.4, 129.3, 127.1, 125.9, 118.0, 115.2, 99.8, 99.6, 67.0, 34.3, 20.6, 14.9. Anal. Calcd for $\mathrm{C}_{23} \mathrm{H}_{21} \mathrm{FN}_{4} \mathrm{O}_{3}: \mathrm{C}, 65.70 ; \mathrm{H}, 5.03 ; \mathrm{N}, 13.33$. Found: $\mathrm{C}, 65.85 ; \mathrm{H}, 4.90 ; \mathrm{N}, 13.21$.

Data for 9c. Yellow solid, yield 55\%, m.p.: 104-105 ${ }^{\circ} \mathrm{C} .{ }^{1} \mathrm{H}-\mathrm{NMR}\left(400 \mathrm{MHz}, \mathrm{CDCl}_{3}\right): \delta 7.82(\mathrm{~s}, 1 \mathrm{H}$, $\mathrm{CH}=\mathrm{N}), 7.66(\mathrm{~d}, J=8.8 \mathrm{~Hz}, 2 \mathrm{H}, \mathrm{Ar}-\mathrm{H}), 7.43$ (d, J = 8.4 Hz, 2H, Ar-H), 6.80-6.85 (m, 4H, Ar-H), 6.46 $\left(\mathrm{s}, 1 \mathrm{H}\right.$, Isoxazole-H), $5.11\left(\mathrm{~s}, 2 \mathrm{H}, \mathrm{CH}_{2}\right), 3.74\left(\mathrm{~s}, 3 \mathrm{H}, \mathrm{OCH}_{3}\right), 3.61\left(\mathrm{~s}, 3 \mathrm{H}, \mathrm{N}-\mathrm{CH}_{3}\right), 2.37\left(\mathrm{~s}, 3 \mathrm{H}, \mathrm{CH}_{3}\right)$. ${ }^{13} \mathrm{C}-\mathrm{NMR}\left(100 \mathrm{MHz} \mathrm{CDCl}_{3}\right): \delta 168.8,162.1,155.8,150.6,148.6,146.9,141.8,136.2,129.3,127.1,125.8$, $119.8,116.4,115.0,114.9,99.5,99.4,67.0,55.6,34.2,14.8$. Anal. Calcd for $\mathrm{C}_{23} \mathrm{H}_{21} \mathrm{FN}_{4} \mathrm{O}_{4}: \mathrm{C}, 63.30 ; \mathrm{H}$, $4.85 ; \mathrm{N}, 12.84$. Found: C, 63.45; H, 4.71; N, 12.96 .

Data for 9d. Yellow solid, yield 47\%, m.p.: $76-78{ }^{\circ} \mathrm{C} .{ }^{1} \mathrm{H}-\mathrm{NMR}\left(400 \mathrm{MHz}, \mathrm{CDCl}_{3}\right): \delta 7.82(\mathrm{~s}, 1 \mathrm{H}, \mathrm{CH}=\mathrm{N})$, $7.68(\mathrm{~d}, J=8.4 \mathrm{~Hz}, 2 \mathrm{H}, \mathrm{Ar}-\mathrm{H}), 7.41-7.44(\mathrm{~m}, 3 \mathrm{H}, \mathrm{Ar}-\mathrm{H}), 7.00-7.15(\mathrm{~m}, 2 \mathrm{H}, \mathrm{Ar}-\mathrm{H}), 6.70(\mathrm{~d}, J=8.4 \mathrm{~Hz}$, 1H, Ar-H), 6.44 (s, 1H, Isoxazole-H), $5.05\left(\mathrm{~s}, 2 \mathrm{H}, \mathrm{CH}_{2}\right), 3.65\left(\mathrm{~s}, 3 \mathrm{H}, \mathrm{CH}_{3}\right), 2.37\left(\mathrm{~s}, 3 \mathrm{H}, \mathrm{CH}_{3}\right) .{ }^{13} \mathrm{C}-\mathrm{NMR}$ 
$\left(100 \mathrm{MHz}_{\mathrm{CDCl}}\right): \delta 168.7,162.0,152.1,147.3,147.1,141.3,136.2,131.0,129.3,128.0,127.1,125.9,124.6$, 122.8, 115.5, 99.7, 99.6, 67.0, 34.3, 14.5. Anal. Calcd for $\mathrm{C}_{22} \mathrm{H}_{18} \mathrm{ClFN}_{4} \mathrm{O}_{3}$ : C, 59.94; $\mathrm{H}, 4.12 ; \mathrm{N}, 12.71$. Found: C, 59.78; H, 4.25; N, 12.85 .

Data for 9e. Yellow oil, yield 50\%. ${ }^{1} \mathrm{H}-\mathrm{NMR}\left(400 \mathrm{MHz}, \mathrm{DMSO}-d_{6}\right): \delta 7.89-7.92(\mathrm{~m}, 2 \mathrm{H}, \mathrm{Ar}-\mathrm{H}), 7.85$ (s, 1H, CH=N), 7.14-7.41 (m, 4H, Ar-H), 6.98-7.01 (m, 2H, Ar-H), 6.86 (s, 1H, Isoxazole-H), 5.02 (s, $2 \mathrm{H}$, $\left.\mathrm{CH}_{2}\right), 3.56\left(\mathrm{~s}, 3 \mathrm{H}, \mathrm{N}-\mathrm{CH}_{3}\right), 2.24\left(\mathrm{~s}, 3 \mathrm{H}, \mathrm{CH}_{3}\right) .{ }^{13} \mathrm{C}-\mathrm{NMR}\left(100 \mathrm{MHz}, \mathrm{CDCl}_{3}\right): \delta 169.1,165.0,162.5,161.9$, 160.0, 157.6, 152.5, 148.8, 148.0, 147.1, 141.3, 127.9, 123.8, 116.7, 116.3, 116.1, 99.8, 98.9, 67.1, 34.4, 14.6. Anal. Calcd for $\mathrm{C}_{22} \mathrm{H}_{18} \mathrm{~F}_{2} \mathrm{~N}_{4} \mathrm{O}_{3}: \mathrm{C}, 62.26 ; \mathrm{H}, 4.27 ; \mathrm{N}, 13.20$. Found: $\mathrm{C}, 62.10 ; \mathrm{H}, 4.41 ; \mathrm{N}, 13.33$.

Data for 9f. White solid, yield 49\%, m.p.: $116-118{ }^{\circ} \mathrm{C} .{ }^{1} \mathrm{H}-\mathrm{NMR}\left(400 \mathrm{MHz}, \mathrm{DMSO}-d_{6}\right): \delta 7.88-7.92(\mathrm{~m}$, $3 \mathrm{H}, \mathrm{CH}=\mathrm{N}$ and Ar-H), 7.36-7.41 (m, 4H, Ar-H), $6.98(\mathrm{~d}, \mathrm{~J}=8.0 \mathrm{~Hz}, 2 \mathrm{H}, \mathrm{Ar}-\mathrm{H}), 6.85$ (s, 1H, Isoxazole-H), $5.02\left(\mathrm{~s}, 2 \mathrm{H}, \mathrm{CH}_{2}\right), 3.56\left(\mathrm{~s}, 3 \mathrm{H}, \mathrm{N}-\mathrm{CH}_{3}\right), 2.24$ (s, 3H, $\left.\mathrm{CH}_{3}\right) .{ }^{13} \mathrm{C}-\mathrm{NMR}\left(100 \mathrm{MHz}, \mathrm{CDCl}_{3}\right): \delta 169.0,165.0$, 162.5, 161.9, 155.2, 147.4, 147.1, 141.3, 129.9, 128.9, 127.9, 127.8, 123.7, 116.7, 116.3, 98.9, 98.6, 67.1, 34.3, 14.5. Anal. Calcd for $\mathrm{C}_{22} \mathrm{H}_{18} \mathrm{ClFN}_{4} \mathrm{O}_{3}$ : C, 59.94; H, 4.12; N, 12.71. Found: C, 60.07; H, 4.02; N, 12.57.

Data for 9g. White solid, yield 52\%, $114-115{ }^{\circ} \mathrm{C}$. ${ }^{1} \mathrm{H}-\mathrm{NMR}\left(400 \mathrm{MHz}, \mathrm{CDCl}_{3}\right): \delta 7.84(\mathrm{~s}, 1 \mathrm{H}, \mathrm{CH}=\mathrm{N})$, 7.72-7.75 (m, 2H, Ar-H), 7.40 (d, J = 9.2 Hz, 2H, Ar-H), $7.16(\mathrm{t}, J=8.8 \mathrm{~Hz}, 2 \mathrm{H}, \mathrm{Ar}-\mathrm{H}), 6.78(\mathrm{~d}, J=8.8 \mathrm{~Hz}$, 2H, Ar-H), 6.40 (s, 1H, Isoxazole-H), 5.08 (s, 2H, $\left.\mathrm{CH}_{2}\right), 3.60\left(\mathrm{~s}, 3 \mathrm{H}, \mathrm{CH}_{3}\right), 2.37\left(\mathrm{~s}, 3 \mathrm{H}, \mathrm{CH}_{3}\right) .{ }^{13} \mathrm{C}-\mathrm{NMR}$ $\left(100 \mathrm{MHz}_{,} \mathrm{CDCl}_{3}\right): \delta 169.0,165.0,162.5,161.9,155.7,147.1,141.3,132.9,127.9,127.8,123.8,117.1,116.3$, 116.2, 116.1, 99.9, 98.9, 67.1, 34.3, 14.6. Anal. Calcd for $\mathrm{C}_{22} \mathrm{H}_{18} \mathrm{BrFN}_{4} \mathrm{O}_{3}$ : C, 54.45; H, 3.74; N, 11.54 . Found: C, 54.30; H, 3.88; N, 11.63.

Data for 9h. White solid, yield 55\%, m.p.: 100-101 ${ }^{\circ} \mathrm{C} .{ }^{1} \mathrm{H}-\mathrm{NMR}\left(400 \mathrm{MHz}, \mathrm{CDCl}_{3}\right): \delta 7.83(\mathrm{~s}, 1 \mathrm{H}$, $\mathrm{CH}=\mathrm{N}), 7.72-7.76(\mathrm{~m}, 2 \mathrm{H}, \mathrm{Ar}-\mathrm{H}), 7.59(\mathrm{~d}, J=8.8 \mathrm{~Hz}, 2 \mathrm{H}, \mathrm{Ar}-\mathrm{H}), 7.16(\mathrm{t}, J=8.8 \mathrm{~Hz}, 2 \mathrm{H}, \mathrm{Ar}-\mathrm{H}), 6.67(\mathrm{~d}$, $J=8.8 \mathrm{~Hz}, 2 \mathrm{H}, \mathrm{Ar}-\mathrm{H}), 6.41\left(\mathrm{~s}, 1 \mathrm{H}\right.$, Isoxazole-H), $5.09\left(\mathrm{~s}, 2 \mathrm{H}, \mathrm{CH}_{2}\right), 3.60\left(\mathrm{~s}, 3 \mathrm{H}, \mathrm{N}-\mathrm{CH}_{3}\right), 2.37\left(\mathrm{~s}, 3 \mathrm{H}, \mathrm{CH}_{3}\right)$. ${ }^{13} \mathrm{C}-\mathrm{NMR}\left(100 \mathrm{MHz}, \mathrm{CDCl}_{3}\right): \delta$ 169.1, 165.0, 162.5, 161.9, 156.6, 147.1, 141.3, 138.8, 127.9, 123.8, 123.7, 117.5, 116.3, 99.9, 98.9, 86.5, 67.1, 34.3, 14.6. Anal. Calcd for $\mathrm{C}_{22} \mathrm{H}_{18} \mathrm{FIN}_{4} \mathrm{O}_{3}$ : C, 49.64; H, 3.41; N, 10.53 . Found: C, 49.52; H, 3.27; N, 10.66 .

Data for 9i. Yellow oil, yield 48\%. ${ }^{1} \mathrm{H}-\mathrm{NMR}\left(400 \mathrm{MHz}, \mathrm{CDCl}_{3}\right): \delta 7.83(\mathrm{~s}, 1 \mathrm{H}, \mathrm{CH}=\mathrm{N}), 7.68(\mathrm{~d}, \mathrm{~J}=8.4 \mathrm{~Hz}$, 2H, Ar-H), 7.43 (d, J = 8.4 Hz, 2H, Ar-H), 6.73-6.94 (m, 3H, Ar-H), 6.46 (s, 1H, Isoxazole-H), 5.05 (s, $2 \mathrm{H}$, $\left.\mathrm{CH}_{2}\right), 3.67\left(\mathrm{~s}, 3 \mathrm{H}, \mathrm{N}-\mathrm{CH}_{3}\right), 2.34\left(\mathrm{~s}, 3 \mathrm{H}, \mathrm{CH}_{3}\right) .{ }^{13} \mathrm{C}-\mathrm{NMR}\left(100 \mathrm{MHz}, \mathrm{CDCl}_{3}\right): \delta 168.9,162.0,147.4,147.2$, 141.1, 136.2, 129.3, 127.1, 125.8, 123.9, 117.5, 117.4, 111.2, 110.9, 105.9, 105.4, 99.4, 99.2, 67.0, 34.3, 14.3. Anal. Calcd for $\mathrm{C}_{22} \mathrm{H}_{17} \mathrm{~F}_{3} \mathrm{~N}_{4} \mathrm{O}_{3}$ : C, 59.73; H, 3.87; N, 12.66. Found: C, 59.85; H, 3.76; N, 12.51.

Data for 9j. Yellow oil, yield 51\%. ${ }^{1} \mathrm{H}-\mathrm{NMR}\left(400 \mathrm{MHz}, \mathrm{CDCl}_{3}\right): \delta 7.83(\mathrm{~s}, 1 \mathrm{H}, \mathrm{CH}=\mathrm{N}), 7.68(\mathrm{~d}, \mathrm{~J}=8.4 \mathrm{~Hz}$, 2H, Ar-H), 7.41-7.44 (m, 3H, Ar-H), 7.08-7.11 (m, 1H, Ar-H), 6.63 (d, J = 8.8 Hz, 1H, Ar-H), 6.44 (s, 1H, Isoxazole-H), $5.05\left(\mathrm{~s}, 2 \mathrm{H}, \mathrm{CH}_{2}\right), 3.64\left(\mathrm{~s}, 3 \mathrm{H}, \mathrm{CH}_{3}\right), 2.35\left(\mathrm{~s}, 3 \mathrm{H}, \mathrm{CH}_{3}\right) .{ }^{13} \mathrm{C}-\mathrm{NMR}\left(100 \mathrm{MHz}, \mathrm{CDCl}_{3}\right): \delta$ 168.9, 161.9, 150.8, 147.3, 146.8, 141.0, 136.2, 130.7, 129.3, 127.9, 127.1, 125.9, 123.7, 121.9, 116.3, 99.8, 99.4, 67.1, 34.3, 14.2. Anal. Calcd for $\mathrm{C}_{22} \mathrm{H}_{17} \mathrm{Cl}_{2} \mathrm{FN}_{4} \mathrm{O}_{3}: \mathrm{C}, 55.59 ; \mathrm{H}, 3.61 ; \mathrm{N}, 11.79$. Found: $\mathrm{C}, 55.45 ; \mathrm{H}, 3.72$; $\mathrm{N}, 11.94$.

Data for 9k. Yellow solid, yield 63\%, m.p.: $113-115{ }^{\circ} \mathrm{C} .{ }^{1} \mathrm{H}-\mathrm{NMR}\left(400 \mathrm{MHz}, \mathrm{CDCl}_{3}\right): \delta 7.82(\mathrm{~s}, 1 \mathrm{H}$, $\mathrm{CH}=\mathrm{N}), 7.67(\mathrm{~d}, J=8.8 \mathrm{~Hz}, 2 \mathrm{H}, \mathrm{Ar}-\mathrm{H}), 7.43$ (d, J = 8.4 Hz, 2H, Ar-H), 6.80-6.85 (m, 4H, Ar-H), 6.46 (s, 1H, Isoxazole-H), $5.11\left(\mathrm{~s}, 2 \mathrm{H}, \mathrm{CH}_{2}\right), 3.74\left(\mathrm{~s}, 3 \mathrm{H}, \mathrm{OCH}_{3}\right), 3.61\left(\mathrm{~s}, 3 \mathrm{H}, \mathrm{N}-\mathrm{CH}_{3}\right), 2.37\left(\mathrm{~s}, 3 \mathrm{H}, \mathrm{CH}_{3}\right)$. ${ }^{13} \mathrm{C}-\mathrm{NMR}\left(100 \mathrm{MHz}, \mathrm{CDCl}_{3}\right): \delta 168.8,162.1,155.9,150.6,148.7,147.0,141.8,136.2,129.3,127.1,125.9$, 116.4, 115.0, 99.6, 99.5, 67.0, 55.7, 34.2, 14.8. Anal. Calcd for $\mathrm{C}_{23} \mathrm{H}_{21} \mathrm{ClN}_{4} \mathrm{O}_{4}$ : C, 61.00; $\mathrm{H}, 4.67 ; \mathrm{N}, 12.37$. Found: C, 60.84; H, 4.81; N, 12.49 .

Data for 91. Yellow solid, yield 61\%, m.p.: 152-154 ${ }^{\circ} \mathrm{C} .{ }^{1} \mathrm{H}-\mathrm{NMR}\left(400 \mathrm{MHz}, \mathrm{CDCl}_{3}\right): \delta 7.85(\mathrm{~s}, 1 \mathrm{H}$, $\mathrm{CH}=\mathrm{N}), 7.68(\mathrm{~d}, J=8.8 \mathrm{~Hz}, 2 \mathrm{H}, \mathrm{Ar}-\mathrm{H}), 7.43(\mathrm{~d}, J=8.4 \mathrm{~Hz}, 2 \mathrm{H}, \mathrm{Ar}-\mathrm{H}), 7.16(\mathrm{~d}, J=8.8 \mathrm{~Hz}, 2 \mathrm{H}, \mathrm{Ar}-\mathrm{H}), 6.91$ $(\mathrm{d}, J=9.2 \mathrm{~Hz}, 2 \mathrm{H}, \mathrm{Ar}-\mathrm{H}), 6.46\left(\mathrm{~s}, 1 \mathrm{H}\right.$, Isoxazole-H), $5.07\left(\mathrm{~s}, 2 \mathrm{H}, \mathrm{CH}_{2}\right), 3.62\left(\mathrm{~s}, 3 \mathrm{H}, \mathrm{N}-\mathrm{CH}_{3}\right), 2.37(\mathrm{~s}, 3 \mathrm{H}$, $\left.\mathrm{CH}_{3}\right) .{ }^{13} \mathrm{C}-\mathrm{NMR}\left(100 \mathrm{MHz}, \mathrm{CDCl}_{3}\right): \delta 168.9,161.9,154.9,147.4,147.2,144.8,141.3,136.3,129.3,127.1$, 
125.8, 122.8, 116.4, 99.9, 99.4, 67.1, 34.3, 14.6. Anal. Calcd for $\mathrm{C}_{23} \mathrm{H}_{18} \mathrm{ClF}_{3} \mathrm{~N}_{4} \mathrm{O}_{4}$ : $\mathrm{C}, 54.50 ; \mathrm{H}, 3.58 ; \mathrm{N}$, 11.05. Found: $\mathrm{C}, 54.63 ; \mathrm{H}, 3.46 ; \mathrm{N}, 11.16$.

Data for $9 \mathrm{~m}$. Yellow solid, yield 56\%, m.p.: $96-98{ }^{\circ} \mathrm{C} .{ }^{1} \mathrm{H}-\mathrm{NMR}\left(400 \mathrm{MHz}, \mathrm{CDCl}_{3}\right): \delta 7.83(\mathrm{~s}, 1 \mathrm{H}, \mathrm{CH}=\mathrm{N})$, $7.68(\mathrm{~d}, J=8.4 \mathrm{~Hz}, 2 \mathrm{H}, \mathrm{Ar}-\mathrm{H}), 7.43(\mathrm{~d}, J=8.4 \mathrm{~Hz}, 2 \mathrm{H}, \mathrm{Ar}-\mathrm{H}), 7.00-7.18(\mathrm{~m}, 3 \mathrm{H}, \mathrm{Ar}-\mathrm{H}), 6.76-6.80(\mathrm{~m}, 1 \mathrm{H}$, $\mathrm{Ar}-\mathrm{H}), 6.45\left(\mathrm{~s}, 1 \mathrm{H}\right.$, Isoxazole-H), $5.05\left(\mathrm{~s}, 2 \mathrm{H}, \mathrm{CH}_{2}\right), 3.67\left(\mathrm{~s}, 3 \mathrm{H}, \mathrm{N}-\mathrm{CH}_{3}\right), 2.36\left(\mathrm{~s}, 3 \mathrm{H}, \mathrm{CH}_{3}\right) .{ }^{13} \mathrm{C}-\mathrm{NMR}$ $\left(100 \mathrm{MHz}, \mathrm{CDCl}_{3}\right): \delta 168.8,162.0,153.2,150.7,147.4,147.1,144.1,141.3,136.2,129.3,127.1,125.8,124.5$, 117.3, 117.1, 116.7, 99.5, 99.4, 67.0, 34.3, 14.4. Anal. Calcd for $\mathrm{C}_{22} \mathrm{H}_{18} \mathrm{ClFN}_{4} \mathrm{O}_{3}: \mathrm{C}, 59.94 ; \mathrm{H}, 4.12 ; \mathrm{N}$, 12.71. Found: C, 59.80; H, 3.99; N, 12.79 .

Data for 9n. White solid, yield 52\%, m.p.: $146-147{ }^{\circ} \mathrm{C} .{ }^{1} \mathrm{H}-\mathrm{NMR}\left(400 \mathrm{MHz}, \mathrm{CDCl}_{3}\right): \delta 7.83(\mathrm{~s}, 1 \mathrm{H}$, $\mathrm{CH}=\mathrm{N}), 7.67(\mathrm{~d}, J=8.4 \mathrm{~Hz}, 2 \mathrm{H}, \mathrm{Ar}-\mathrm{H}), 7.44(\mathrm{~d}, J=8.8 \mathrm{~Hz}, 2 \mathrm{H}, \mathrm{Ar}-\mathrm{H}), 6.83-7.00(\mathrm{~m}, 4 \mathrm{H}, \mathrm{Ar}-\mathrm{H}), 6.44$ $(\mathrm{d}, 1 \mathrm{H}$, Isoxazole- $\mathrm{H}), 5.09\left(\mathrm{~s}, 2 \mathrm{H}, \mathrm{CH}_{2}\right), 3.61\left(\mathrm{~s}, 3 \mathrm{H}, \mathrm{N}-\mathrm{CH}_{3}\right), 2.36\left(\mathrm{~s}, 3 \mathrm{H}, \mathrm{CH}_{3}\right) .{ }^{13} \mathrm{C}-\mathrm{NMR}(100 \mathrm{MHz}$, $\left.\mathrm{CDCl}_{3}\right): \delta 168.8,162.0,159.9,157.5,152.6,147.9,147.1,141.5,136.2,129.3,127.1,125.8,116.6,116.4,99.7$, 99.4, 67.0, 34.2, 14.6. Anal. Calcd for $\mathrm{C}_{22} \mathrm{H}_{18} \mathrm{ClFN}_{4} \mathrm{O}_{3}$ : C, 59.94; $\mathrm{H}, 4.12 ; \mathrm{N}, 12.71$. Found: $\mathrm{C}, 60.04 ; \mathrm{H}$, $4.23 ; \mathrm{N}, 12.57$.

Data for 9o. White solid, yield 50\%, m.p.: $116-118{ }^{\circ} \mathrm{C} .{ }^{1} \mathrm{H}-\mathrm{NMR}\left(400 \mathrm{MHz}, \mathrm{CDCl}_{3}\right): \delta 7.85(\mathrm{~s}, 1 \mathrm{H}$, $\mathrm{CH}=\mathrm{N}), 7.68(\mathrm{~d}, J=8.4 \mathrm{~Hz}, 2 \mathrm{H}, \mathrm{Ar}-\mathrm{H}), 7.44(\mathrm{~d}, J=8.8 \mathrm{~Hz}, 2 \mathrm{H}, \mathrm{Ar}-\mathrm{H}), 6.82-7.20(\mathrm{~m}, 4 \mathrm{H}, \mathrm{Ar}-\mathrm{H}), 6.42$ (s, 1H, Isoxazole-H), $5.09\left(\mathrm{~s}, 2 \mathrm{H}, \mathrm{CH}_{2}\right), 3.61\left(\mathrm{~s}, 3 \mathrm{H}, \mathrm{N}-\mathrm{CH}_{3}\right), 2.37\left(\mathrm{~s}, 3 \mathrm{H}, \mathrm{CH}_{3}\right) .{ }^{13} \mathrm{C}-\mathrm{NMR}(100 \mathrm{MHz}$, $\left.\mathrm{CDCl}_{3}\right): \delta 168.8,162.0,157.1,147.2,141.3,136.2,131.1,129.3,127.1,126.9,125.8,123.1,118.7,114.1,100.0$, 99.4, 67.1, 34.3, 14.5. Anal. Calcd for $\mathrm{C}_{22} \mathrm{H}_{18} \mathrm{BrClN}_{4} \mathrm{O}_{3}$ : C, 52.66; $\mathrm{H}, 3.62 ; \mathrm{N}, 11.17$. Found: $\mathrm{C}, 52.79 ; \mathrm{H}$, $3.48 ; \mathrm{N}, 11.30$.

Data for 9p. White solid, yield 58\%, m.p.: $147-149{ }^{\circ} \mathrm{C} .{ }^{1} \mathrm{H}-\mathrm{NMR}\left(400 \mathrm{MHz}, \mathrm{CDCl}_{3}\right): \delta 7.83(\mathrm{~s}, 1 \mathrm{H}$, $\mathrm{CH}=\mathrm{N}), 7.68(\mathrm{~d}, J=8.4 \mathrm{~Hz}, 2 \mathrm{H}, \mathrm{Ar}-\mathrm{H}), 7.59(\mathrm{~d}, J=8.8 \mathrm{~Hz}, 2 \mathrm{H}, \mathrm{Ar}-\mathrm{H}), 7.45(\mathrm{~d}, J=8.4 \mathrm{~Hz}, 2 \mathrm{H}, \mathrm{Ar}-\mathrm{H})$, $6.67(\mathrm{~d}, J=8.8 \mathrm{~Hz}, 2 \mathrm{H}, \mathrm{Ar}-\mathrm{H}), 6.45\left(\mathrm{~s}, 1 \mathrm{H}\right.$, Isoxazole-H), $5.09\left(\mathrm{~s}, 2 \mathrm{H}, \mathrm{CH}_{2}\right), 3.59\left(\mathrm{~s}, 3 \mathrm{H}, \mathrm{CH}_{3}\right), 2.36(\mathrm{~s}, 3 \mathrm{H}$, $\left.\mathrm{CH}_{3}\right) .{ }^{13} \mathrm{C}-\mathrm{NMR}\left(100 \mathrm{MHz}, \mathrm{CDCl}_{3}\right): \delta 168.9,161.9,156.6,147.2,147.1,141.3,138.8,136.2,129.3,127.1$, 125.8, 117.5, 99.9, 99.4, 86.5, 67.1, 34.3, 14.6. Anal. Calcd for $\mathrm{C}_{22} \mathrm{H}_{18} \mathrm{ClIN}_{4} \mathrm{O}_{3}: \mathrm{C}, 48.15 ; \mathrm{H}, 3.31 ; \mathrm{N}, 10.21$. Found: $\mathrm{C}, 48.01 ; \mathrm{H}, 3.25 ; \mathrm{N}, 10.33$.

Data for 9q. Yellow solid, yield 49\%, m.p.: 80-81 ${ }^{\circ} \mathrm{C} .{ }^{1} \mathrm{H}-\mathrm{NMR}\left(400 \mathrm{MHz}, \mathrm{CDCl}_{3}\right): \delta 7.83(\mathrm{~s}, 1 \mathrm{H}, \mathrm{CH}=\mathrm{N})$, $7.68(\mathrm{~d}, J=8.4 \mathrm{~Hz}, 2 \mathrm{H}, \mathrm{Ar}-\mathrm{H}), 7.44(\mathrm{~d}, J=8.4 \mathrm{~Hz}, 2 \mathrm{H}, \mathrm{Ar}-\mathrm{H}), 6.70-6.95(\mathrm{~m}, 3 \mathrm{H}, \mathrm{Ar}-\mathrm{H}), 6.46(\mathrm{~s}, 1 \mathrm{H}$, Isoxazole-H), $5.06\left(\mathrm{~s}, 2 \mathrm{H}, \mathrm{CH}_{2}\right), 3.68\left(\mathrm{~s}, 3 \mathrm{H}, \mathrm{CH}_{3}\right), 2.34\left(\mathrm{~s}, 3 \mathrm{H}, \mathrm{CH}_{3}\right) .{ }^{13} \mathrm{C}-\mathrm{NMR}\left(100 \mathrm{MHz}, \mathrm{CDCl}_{3}\right): \delta$ 167.8, 160.9, 158.5, 156.0, 152.1, 149.6, 146.4, 146.2, 140.1, 139.6, 135.2, 128.3, 126.0, 124.8, 116.5, 110.1, 104.8, 98.3, 98.2, 66.0, 33.2, 13.2. Anal. Calcd for $\mathrm{C}_{22} \mathrm{H}_{17} \mathrm{ClF}_{2} \mathrm{~N}_{4} \mathrm{O}_{3}: \mathrm{C}, 57.59 ; \mathrm{H}, 3.73 ; \mathrm{N}, 12.21$. Found: $\mathrm{C}$, $57.75 ; \mathrm{H}, 3.58 ; \mathrm{N}, 12.08$.

Data for 9r. White solid, yield 61\%, m.p.: 86-88 ${ }^{\circ} \mathrm{C} .{ }^{1} \mathrm{H}-\mathrm{NMR}\left(400 \mathrm{MHz}, \mathrm{CDCl}_{3}\right): \delta 7.91-7.97(\mathrm{~m}, 1 \mathrm{H}$, $\mathrm{Ar}-\mathrm{H}), 7.81(\mathrm{~s}, 1 \mathrm{H}, \mathrm{CH}=\mathrm{N}), 6.67-7.04\left(\mathrm{~m}, 7 \mathrm{H}, \mathrm{Ar}-\mathrm{H}\right.$ and Isoxazole-H), $5.12\left(\mathrm{~s}, 2 \mathrm{H}, \mathrm{CH}_{2}\right), 3.75(\mathrm{~s}, 3 \mathrm{H}$, $\left.\mathrm{OCH}_{3}\right), 3.61\left(\mathrm{~s}, 3 \mathrm{H}, \mathrm{N}-\mathrm{CH}_{3}\right), 2.38\left(\mathrm{~s}, 3 \mathrm{H}, \mathrm{CH}_{3}\right) .{ }^{13} \mathrm{C}-\mathrm{NMR}\left(100 \mathrm{MHz}, \mathrm{CDCl}_{3}\right): \delta 165.1,162.9,162.2,160.7$, 158.3, 155.9, 150.6, 148.8, 147.0, 141.8, 128.9, 119.8, 116.4, 115.0, 112.4, 104.8, 102.9, 99.5, 66.9, 55.7, 34.2, 14.8. Anal. Calcd for $\mathrm{C}_{23} \mathrm{H}_{20} \mathrm{~F}_{2} \mathrm{~N}_{4} \mathrm{O}_{4}$ : $\mathrm{C}, 60.79 ; \mathrm{H}, 4.44 ; \mathrm{N}, 12.33$. Found: $\mathrm{C}, 60.65 ; \mathrm{H}, 4.59 ; \mathrm{N}, 12.45$.

Data for 9s. Yellow solid, yield 52\%, m.p.: $106-108^{\circ} \mathrm{C} .{ }^{1} \mathrm{H}-\mathrm{NMR}\left(400 \mathrm{MHz}, \mathrm{CDCl}_{3}\right): \delta 7.91-7.97(\mathrm{~m}, 1 \mathrm{H}$, Ar-H), $7.83(\mathrm{~s}, 1 \mathrm{H}, \mathrm{CH}=\mathrm{N}), 7.10(\mathrm{~d}, J=6.0 \mathrm{~Hz}, 2 \mathrm{H}, \mathrm{Ar}-\mathrm{H}), 6.63-7.03(\mathrm{~m}, 5 \mathrm{H}, \mathrm{Ar}-\mathrm{H}$ and Isoxazole-H), 5.10 $\left(\mathrm{s}, 2 \mathrm{H}, \mathrm{CH}_{2}\right), 3.61\left(\mathrm{~s}, 3 \mathrm{H}, \mathrm{CH}_{3}\right), 2.37\left(\mathrm{~s}, 3 \mathrm{H}, \mathrm{CH}_{3}\right) .{ }^{13} \mathrm{C}-\mathrm{NMR}\left(100 \mathrm{MHz}, \mathrm{CDCl}_{3}\right): \delta 165.0,162.9,162.1$, $160.8,160.0,158.2,157.5,152.6,147.9,147.1,141.5,128.9,116.6,116.4,112.4,104.8,102.8,99.7,67.0,34.2$, 14.6. Anal. Calcd for $\mathrm{C}_{22} \mathrm{H}_{17} \mathrm{~F}_{3} \mathrm{~N}_{4} \mathrm{O}_{3}: \mathrm{C}, 59.73 ; \mathrm{H}, 3.87 ; \mathrm{N}, 12.66$. Found: $\mathrm{C}, 59.58 ; \mathrm{H}, 4.01 ; \mathrm{N}, 12.78$.

Data for 9 t. Yellow solid, yield 56\%, m.p.: $108-109{ }^{\circ} \mathrm{C} .{ }^{1} \mathrm{H}-\mathrm{NMR}\left(400 \mathrm{MHz}, \mathrm{CDCl}_{3}\right): \delta 7.92-7.97(\mathrm{~m}, 1 \mathrm{H}$, $\mathrm{Ar}-\mathrm{H}), 7.84(\mathrm{~s}, 1 \mathrm{H}, \mathrm{CH}=\mathrm{N}), 6.63-7.26\left(\mathrm{~m}, 7 \mathrm{H}, \mathrm{Ar}-\mathrm{H}\right.$ and Isoxazole-H), $5.10\left(\mathrm{~s}, 2 \mathrm{H}, \mathrm{CH}_{2}\right), 3.61(\mathrm{~s}, 3 \mathrm{H}$, $\left.\mathrm{N}-\mathrm{CH}_{3}\right), 2.37$ (s, 3H, $\left.\mathrm{CH}_{3}\right) \cdot{ }^{13} \mathrm{C}-\mathrm{NMR}\left(100 \mathrm{MHz}, \mathrm{CDCl}_{3}\right): \delta 165.0,163.0,162.6,162.1,160.7,158.2,155.1$, 147.4, 147.2, 141.4, 129.9, 128.8, 116.6, 112.4, 112.2, 104.8, 102.8, 99.9, 67.0, 34.3, 14.5. Anal. Calcd for $\mathrm{C}_{22} \mathrm{H}_{17} \mathrm{ClF}_{2} \mathrm{~N}_{4} \mathrm{O}_{3}: \mathrm{C}, 57.59 ; \mathrm{H}, 3.73 ; \mathrm{N}, 12.21$. Found: $\mathrm{C}, 57.49 ; \mathrm{H}, 3.86 ; \mathrm{N}, 12.35$. 
Data for 9 u. Yellow solid, yield 60\%, m.p.: $101-103{ }^{\circ} \mathrm{C} .{ }^{1} \mathrm{H}-\mathrm{NMR}\left(400 \mathrm{MHz}, \mathrm{CDCl}_{3}\right): \delta 7.89(\mathrm{~d}, \mathrm{~J}=8.4 \mathrm{~Hz}$, $1 \mathrm{H}, \mathrm{Ar}-\mathrm{H}), 7.84(\mathrm{~s}, 1 \mathrm{H}, \mathrm{CH}=\mathrm{N}), 7.53(\mathrm{~d}, \mathrm{~J}=2.0 \mathrm{~Hz}, 1 \mathrm{H}, \mathrm{Ar}-\mathrm{H}), 6.76-7.40(\mathrm{~m}, 6 \mathrm{H}, \mathrm{Ar}-\mathrm{H}$ and Isoxazole-H), $5.11\left(\mathrm{~s}, 2 \mathrm{H}, \mathrm{CH}_{2}\right), 3.60$ (s, 3H, N-CH 3$), 2.37$ (s, 3H, $\left.\mathrm{CH}_{3}\right) .{ }^{13} \mathrm{C}-\mathrm{NMR}\left(100 \mathrm{MHz}, \mathrm{CDCl}_{3}\right): \delta 165.2,161.8$, 155.7, 147.3, 147.1, 141.4, 136.2, 132.9, 132.2, 130.6, 130.1, 127.7, 124.7, 117.0, 116.2, 104.3, 99.8, 67.0, 34.3, 14.6. Anal. Calcd for $\mathrm{C}_{22} \mathrm{H}_{17} \mathrm{BrF}_{2} \mathrm{~N}_{4} \mathrm{O}_{3}$ : C, 52.50; H, 3.40; N, 11.13. Found: C, 52.66; H, 3.27; N, 11.27.

Data for 9v. Yellow solid, yield 58\%, m.p.: 104-106 ${ }^{\circ} \mathrm{C} .{ }^{1} \mathrm{H}-\mathrm{NMR}\left(400 \mathrm{MHz}, \mathrm{CDCl}_{3}\right): \delta 7.89(\mathrm{~d}, \mathrm{~J}=8.8 \mathrm{~Hz}$, $1 \mathrm{H}, \mathrm{Ar}-\mathrm{H}), 7.83(\mathrm{~s}, 1 \mathrm{H}, \mathrm{CH}=\mathrm{N}), 7.58(\mathrm{~d}, \mathrm{~J}=8.4 \mathrm{~Hz}, 2 \mathrm{H}, \mathrm{Ar}-\mathrm{H}), 6.65-7.54(\mathrm{~m}, 5 \mathrm{H}, \mathrm{Ar}-\mathrm{H}$ and Isoxazole-H), $5.11\left(\mathrm{~s}, 2 \mathrm{H}, \mathrm{CH}_{2}\right), 3.60\left(\mathrm{~s}, 3 \mathrm{H}, \mathrm{CH}_{3}\right), 2.38\left(\mathrm{~s}, 3 \mathrm{H}, \mathrm{CH}_{3}\right) .{ }^{13} \mathrm{C}-\mathrm{NMR}\left(100 \mathrm{MHz} \mathrm{CDCl}_{3}\right): \delta 165.2,161.8$, 156.5, 147.3, 147.1, 141.4, 138.8, 136.3, 132.3, 130.7, 130.1, 127.7, 124.7, 117.5, 104.3, 99.9, 86.6, 67.0, 34.3, 14.6. Anal. Calcd for $\mathrm{C}_{22} \mathrm{H}_{17} \mathrm{~F}_{2} \mathrm{IN}_{4} \mathrm{O}_{3}$ : C, 48.02; H, 3.11; N, 10.18. Found: C, 48.13; H, 3.01; N, 10.33.

\subsubsection{General Procedure for the Preparation of $\mathbf{1 0}$}

To a mixture of intermediate 8 (10 $\mathrm{mmol})$, 4-hydroxybenzaldehyde $(12 \mathrm{mmol})$ in acetonitrile $(60 \mathrm{~mL})$ at room temperature, was added cesium carbonate $(13 \mathrm{mmol})$. The resulting mixture was heated to reflux for $5 \mathrm{~h}$. After being cooled to room temperature, the mixture was poured into water $(100 \mathrm{~mL})$, and the solid precipitate was filtered, washed with water, and dried to afford corresponding compound 10, with yields ranging from $71 \%$ to $77 \%$, which could be used for the next reaction without further purification.

\subsubsection{General Procedure for the Preparation of $\mathbf{1 1}$}

To a well stirred cold $\left(0^{\circ} \mathrm{C}\right)$ solution of intermediate $10(4 \mathrm{mmol})$ and THF $(60 \mathrm{~mL})$, was added $\mathrm{LiAlH}_{4}(6 \mathrm{mmol})$ in three portions and the reaction mixture was stirred at $0{ }^{\circ} \mathrm{C}$ for $30 \mathrm{~min}$. To the above solution, was added ice water $(40 \mathrm{~mL})$. After the solid precipitate was filtered, the filtrate was extracted with ethyl acetate $(3 \times 30 \mathrm{~mL})$. The combined extracts were dried over anhydrous sodium sulfate, filtered, and concentrated to give compound 11 with yields ranging from $68 \%$ to $72 \%$, which could be used for the next reaction without further purification.

\subsubsection{General Procedure for the Preparation of $\mathbf{1 2}$}

To a well stirred cold $\left(0^{\circ} \mathrm{C}\right)$ solution of compound $11(4 \mathrm{mmol})$ in $\mathrm{CH}_{2} \mathrm{Cl}_{2}(50 \mathrm{~mL})$, was added dropwise a mixture of thionyl chloride $(8 \mathrm{mmol})$ in $\mathrm{CH}_{2} \mathrm{Cl}_{2}(10 \mathrm{~mL})$. Then, several drops of DMF was added thereto. The resulting mixture was stirred at $0{ }^{\circ} \mathrm{C}$ for $6-8 \mathrm{~h}$. To the above solution, was added ice water $(50 \mathrm{~mL})$, and the $\mathrm{pH}$ value of the mixture was adjusted to 6 by saturated sodium bicarbonate solution. The organic layer was dried over anhydrous sodium sulfate, filtered, and concentrated to afford the corresponding compound 12, with yields ranging from $62 \%$ to $65 \%$, which could be used for the following transformations without further purification.

\subsubsection{General Procedure for the Preparation of 13a-13f}

To a mixture of intermediate $12(4 \mathrm{mmol})$, compound $3(5 \mathrm{mmol})$, and potassium carbonate $(10 \mathrm{mmol})$ in acetonitrile $(30 \mathrm{~mL})$ at room temperature. The resulting mixture was heated to reflux for 10-15 h. The reaction mixture was allowed to cool at room temperature and filtered. The solvent was evaporated under reduced pressure, and the residue was admixed with water $(40 \mathrm{~mL})$ and extracted with ethyl acetate $(3 \times 30 \mathrm{~mL})$. The combined organic layer was washed with water $(3 \times 20 \mathrm{~mL})$, dried over anhydrous sodium sulfate, filtered, and concentrated. The residue was purified by silica gel column chromatography using a mixture of petroleum ether and ethyl acetate as an eluent to produce the title compounds 13a-13f, with yields ranging from 46\% to 56\%. Pyrazole oxime derivatives 13a-13f were novel and the physical and spectral data for these compounds are listed below. ${ }^{1} \mathrm{H}-\mathrm{NMR}$ and ${ }^{13} \mathrm{C}-\mathrm{NMR}$ spectra are provided in the Supplementary Materials. 
Data for 13a. White solid, yield 50\%, m.p.: $77-79{ }^{\circ} \mathrm{C} .{ }^{1} \mathrm{H}-\mathrm{NMR}\left(400 \mathrm{MHz}, \mathrm{CDCl}_{3}\right): \delta 7.75-7.78(\mathrm{~m}, 3 \mathrm{H}$, Ar-H and $\mathrm{CH}=\mathrm{N}), 7.07-7.26(\mathrm{~m}, 6 \mathrm{H}, \mathrm{Ar}-\mathrm{H}), 6.94(\mathrm{~d}, J=8.0 \mathrm{~Hz}, 2 \mathrm{H}, \mathrm{Ar}-\mathrm{H}), 6.76(\mathrm{~d}, J=8.0 \mathrm{~Hz}, 2 \mathrm{H}, \mathrm{Ar}-\mathrm{H})$, 6.58 (s, 1H, Isoxazole-H), $5.18\left(\mathrm{~s}, 2 \mathrm{H}, \mathrm{CH}_{2}\right), 4.94\left(\mathrm{~s}, 2 \mathrm{H}, \mathrm{CH}_{2}\right), 3.58\left(\mathrm{~s}, 3 \mathrm{H}, \mathrm{N}-\mathrm{CH}_{3}\right), 2.37\left(\mathrm{~s}, 3 \mathrm{H}, \mathrm{CH}_{3}\right)$, $2.30\left(\mathrm{~s}, 3 \mathrm{H}, \mathrm{CH}_{3}\right) .{ }^{13} \mathrm{C}-\mathrm{NMR}\left(100 \mathrm{MHz}, \mathrm{CDCl}_{3}\right): \delta 169.6,165.1,162.6,161.5,157.8,154.7,148.1,146.8$, 140.7, 133.1, 130.8, 130.4, 128.0, 127.9, 123.6, 116.4, 116.1, 115.1, 114.6, 100.2, 98.6, 75.6, 61.8, 34.2, 20.6, 14.9. Anal. Calcd for $\mathrm{C}_{30} \mathrm{H}_{27} \mathrm{FN}_{4} \mathrm{O}_{4}$ : C, 68.43; H, 5.17; N, 10.64. Found: $\mathrm{C}, 68.59 ; \mathrm{H}, 5.05 ; \mathrm{N}, 10.77$.

Data for 13b. White solid, yield 48\%, m.p.: $99-101{ }^{\circ} \mathrm{C} .{ }^{1} \mathrm{H}-\mathrm{NMR}\left(400 \mathrm{MHz}, \mathrm{CDCl}_{3}\right): \delta 7.75-7.78(\mathrm{~m}, 3 \mathrm{H}$, Ar-H and $\mathrm{CH}=\mathrm{N}), 7.13-7.24(\mathrm{~m}, 4 \mathrm{H}, \mathrm{Ar}-\mathrm{H}), 6.82-7.00(\mathrm{~m}, 6 \mathrm{H}, \mathrm{Ar}-\mathrm{H}), 6.59(\mathrm{~s}, 1 \mathrm{H}$, Isoxazole-H), 5.18 (s, $\left.2 \mathrm{H}, \mathrm{CH}_{2}\right), 4.92\left(\mathrm{~s}, 2 \mathrm{H}, \mathrm{CH}_{2}\right), 3.60\left(\mathrm{~s}, 3 \mathrm{H}, \mathrm{N}-\mathrm{CH}_{3}\right), 2.35\left(\mathrm{~s}, 3 \mathrm{H}, \mathrm{CH}_{3}\right) .{ }^{13} \mathrm{C}-\mathrm{NMR}\left(100 \mathrm{MHz}, \mathrm{CDCl}_{3}\right): \delta$ 169.6, 165.1, 162.6, 161.5, 160.0, 157.8, 157.5, 152.7, 152.6, 147.7, 147.0, 140.4, 130.7, 130.3, 128.0, 127.9, 123.6, 116.6, 116.5, 116.4, 116.1, 114.6, 100.2, 98.6, 75.7, 61.8, 34.2, 14.6. Anal. Calcd for $\mathrm{C}_{29} \mathrm{H}_{24} \mathrm{~F}_{2} \mathrm{~N}_{4} \mathrm{O}_{4}$ : C, 65.65; H, 4.56; N, 10.56. Found: C, 65.78; H, 4.42; N, 10.65 .

Data for 13c. White solid, yield 46\%, m.p.: $91-93{ }^{\circ} \mathrm{C} .{ }^{1} \mathrm{H}-\mathrm{NMR}\left(400 \mathrm{MHz}, \mathrm{CDCl}_{3}\right): \delta 7.75-7.78(\mathrm{~m}, 3 \mathrm{H}$, Ar-H and $\mathrm{CH}=\mathrm{N}), 7.40(\mathrm{~d}, J=8.0 \mathrm{~Hz}, 2 \mathrm{H}, \mathrm{Ar}-\mathrm{H}), 7.13-7.22(\mathrm{~m}, 4 \mathrm{H}, \mathrm{Ar}-\mathrm{H}), 6.94(\mathrm{~d}, J=8.0 \mathrm{~Hz}, 2 \mathrm{H}, \mathrm{Ar}-\mathrm{H})$, $6.76(\mathrm{~d}, J=8.0 \mathrm{~Hz}, 2 \mathrm{H}, \mathrm{Ar}-\mathrm{H}), 6.59\left(\mathrm{~s}, 1 \mathrm{H}\right.$, Isoxazole-H), $5.18\left(\mathrm{~s}, 2 \mathrm{H}, \mathrm{CH}_{2}\right), 4.91\left(\mathrm{~s}, 2 \mathrm{H}, \mathrm{CH}_{2}\right), 3.59(\mathrm{~s}, 3 \mathrm{H}$, $\left.\mathrm{N}-\mathrm{CH}_{3}\right), 2.34\left(\mathrm{~s}, 3 \mathrm{H}, \mathrm{CH}_{3}\right) .{ }^{13} \mathrm{C}-\mathrm{NMR}\left(100 \mathrm{MHz}, \mathrm{CDCl}_{3}\right): \delta 169.6,165.1,162.6,161.5,157.8,155.8,147.0$, 140.3, 132.9, 130.7, 130.3, 128.0, 127.9, 123.6, 117.1, 116.4, 116.1, 114.6, 100.3, 98.6, 75.7, 61.8, 34.2, 14.5 . Anal. Calcd for $\mathrm{C}_{29} \mathrm{H}_{24} \mathrm{BrFN}_{4} \mathrm{O}_{4}$ : C, 58.89; H, 4.09; N, 9.47. Found: C, 58.74; H, 4.20; N, 9.61.

Data for 13d. White solid, yield 53\%, m.p.: $97-99{ }^{\circ} \mathrm{C} .{ }^{1} \mathrm{H}-\mathrm{NMR}\left(400 \mathrm{MHz}, \mathrm{CDCl}_{3}\right): \delta 7.75-7.78(\mathrm{~m}, 3 \mathrm{H}$, Ar-H and $\mathrm{CH}=\mathrm{N}), 7.59(\mathrm{~d}, J=8.0 \mathrm{~Hz}, 2 \mathrm{H}, \mathrm{Ar}-\mathrm{H}), 7.13-7.22(\mathrm{~m}, 4 \mathrm{H}, \mathrm{Ar}-\mathrm{H}), 6.95(\mathrm{~d}, J=8.0 \mathrm{~Hz}, 2 \mathrm{H}, \mathrm{Ar}-\mathrm{H})$, $6.65(\mathrm{~d}, J=8.0 \mathrm{~Hz}, 2 \mathrm{H}, \mathrm{Ar}-\mathrm{H}), 6.59\left(\mathrm{~s}, 1 \mathrm{H}\right.$, Isoxazole-H), $5.19\left(\mathrm{~s}, 2 \mathrm{H}, \mathrm{CH}_{2}\right), 4.91\left(\mathrm{~s}, 2 \mathrm{H}, \mathrm{CH}_{2}\right), 3.58(\mathrm{~s}, 3 \mathrm{H}$, $\left.\mathrm{N}-\mathrm{CH}_{3}\right), 2.34\left(\mathrm{~s}, 3 \mathrm{H}, \mathrm{CH}_{3}\right) .{ }^{13} \mathrm{C}-\mathrm{NMR}\left(100 \mathrm{MHz}, \mathrm{CDCl}_{3}\right): \delta 169.5,165.1,162.6,161.5,157.8,156.6,147.0$, 146.9, 140.2, 138.8, 130.7, 130.3, 128.0, 127.9, 123.6, 117.5, 116.4, 116.1, 114.6, 100.3, 98.6, 86.4, 75.7, 61.8, 34.2, 14.5. Anal. Calcd for $\mathrm{C}_{29} \mathrm{H}_{24} \mathrm{FIN}_{4} \mathrm{O}_{4}$ : C, 54.56; H, 3.79; N, 8.78. Found: C, 54.69; H, 3.90; N, 8.64.

Data for 13e. White solid, yield 51\%, m.p.: $88-90{ }^{\circ} \mathrm{C} .{ }^{1} \mathrm{H}-\mathrm{NMR}\left(400 \mathrm{MHz}, \mathrm{CDCl}_{3}\right): \delta 7.77(\mathrm{~s}, 1 \mathrm{H}, \mathrm{CH}=\mathrm{N})$, $7.70(\mathrm{~d}, J=8.0 \mathrm{~Hz}, 2 \mathrm{H}, \mathrm{Ar}-\mathrm{H}), 7.39-7.44(\mathrm{~m}, 4 \mathrm{H}, \mathrm{Ar}-\mathrm{H}), 7.21(\mathrm{~d}, J=8.0 \mathrm{~Hz}, 2 \mathrm{H}, \mathrm{Ar}-\mathrm{H}), 6.94(\mathrm{~d}, J=8.0 \mathrm{~Hz}$, $2 \mathrm{H}, \mathrm{Ar}-\mathrm{H}), 6.76(\mathrm{~d}, J=8.0 \mathrm{~Hz}, 2 \mathrm{H}, \mathrm{Ar}-\mathrm{H}), 6.63\left(\mathrm{~s}, 1 \mathrm{H}\right.$, Isoxazole-H), $5.19\left(\mathrm{~s}, 2 \mathrm{H}, \mathrm{CH}_{2}\right), 4.91\left(\mathrm{~s}, 2 \mathrm{H}, \mathrm{CH}_{2}\right)$, $3.59\left(\mathrm{~s}, 3 \mathrm{H}, \mathrm{N}-\mathrm{CH}_{3}\right), 2.34\left(\mathrm{~s}, 3 \mathrm{H}, \mathrm{CH}_{3}\right) .{ }^{13} \mathrm{C}-\mathrm{NMR}\left(100 \mathrm{MHz}, \mathrm{CDCl}_{3}\right): \delta 169.4,161.6,157.8,155.8,147.0$, 140.2, 136.4, 133.3, 132.9, 130.7, 130.3, 129.3, 127.1, 125.7, 119.4, 117.1, 116.1, 114.6, 100.3, 99.1, 75.7, 61.7, 34.2, 14.5. Anal. Calcd for $\mathrm{C}_{29} \mathrm{H}_{24} \mathrm{BrClN}_{4} \mathrm{O}_{4}: \mathrm{C}, 57.30 ; \mathrm{H}, 3.98 ; \mathrm{N}, 9.22$. Found: $\mathrm{C}, 57.14 ; \mathrm{H}, 3.85 ; \mathrm{N}, 9.36$.

Data for 13f. White solid, yield 56\%, m.p.: $83-85^{\circ} \mathrm{C} .{ }^{1} \mathrm{H}-\mathrm{NMR}\left(400 \mathrm{MHz}, \mathrm{CDCl}_{3}\right): \delta 7.76(\mathrm{~s}, 1 \mathrm{H}, \mathrm{CH}=\mathrm{N})$, $7.71(\mathrm{~d}, J=8.0 \mathrm{~Hz}, 2 \mathrm{H}, \mathrm{Ar}-\mathrm{H}), 7.59(\mathrm{~d}, J=8.0 \mathrm{~Hz}, 2 \mathrm{H}, \mathrm{Ar}-\mathrm{H}), 7.44(\mathrm{~d}, J=8.0 \mathrm{~Hz}, 2 \mathrm{H}, \mathrm{Ar}-\mathrm{H}), 7.21(\mathrm{~d}$, $J=8.0 \mathrm{~Hz}, 2 \mathrm{H}, \mathrm{Ar}-\mathrm{H}), 6.63-6.96(\mathrm{~m}, 5 \mathrm{H}, \mathrm{Ar}-\mathrm{H}$ and Isoxazole- $\mathrm{H}), 5.19\left(\mathrm{~s}, 2 \mathrm{H}, \mathrm{CH}_{2}\right), 4.91\left(\mathrm{~s}, 2 \mathrm{H}, \mathrm{CH}_{2}\right)$, $3.59\left(\mathrm{~s}, 3 \mathrm{H}, \mathrm{N}-\mathrm{CH}_{3}\right), 2.34\left(\mathrm{~s}, 3 \mathrm{H}, \mathrm{CH}_{3}\right) .{ }^{13} \mathrm{C}-\mathrm{NMR}\left(100 \mathrm{MHz} \mathrm{CDCl}_{3}\right): \delta 169.4,161.6,157.8,156.6,147.0$, 146.9, 140.2, 139.2, 138.8, 136.4, 130.7, 130.4, 129.3, 127.1, 119.6, 117.6, 114.6, 100.4, 99.2, 86.4, 75.7, 61.8, 34.2, 14.5. Anal. Calcd for $\mathrm{C}_{29} \mathrm{H}_{24} \mathrm{ClIN}_{4} \mathrm{O}_{4}$ : C, 53.19; H, 3.69; N, 8.56. Found: C, 53.33; H, 3.54; N, 8.47.

\subsection{Biological Tests}

\subsubsection{Bioassay Methods}

All of the bioassays were performed on representative test organisms reared in the laboratory. The bioassay was repeated in triplicate. Acaricidal and insecticidal assessments were made on a dead/alive basis, and mortality rates were corrected using Abbott's formula.

\subsubsection{Insecticidal Activities against Mythimna separata}

The larvicidal activities of the title compounds against Mythimna separata were tested by foliar application [29]. Corn leaves were dipped into the obtained solutions for 2-3 s. After air-drying, the soaked leaves were put into a culture dish with a piece of filter paper, followed by inoculation 
of 10 third-instar M. separata larvae per dish. Covered with gauze and kept in observation room for normal cultivation at $24^{\circ} \mathrm{C}-27^{\circ} \mathrm{C}$. Mortality was assessed $48 \mathrm{~h}$ after treatment. The individuals who did not respond to the touch of writing brush were recognized as dead. Each test was run three times and the results were averaged. Pyridalyl, as the control compound, was tested under the same conditions.

3.2.3. Acaricidal Activities against Tetranychus cinnabarinus, and Insecticidal Activities against Aphis medicaginis and Nilaparvata lugens

The acaricidal activities against Tetranychus cinnabarinus, and insecticidal activities against Aphis medicaginis and Nilaparvata lugens of the title compounds were tested by the spray method [30]. Under the Potter spray tower, horsebean leaves, inoculated with T. cinnabarinus were separately treated with solutions of tested compounds. After that, the resultant horsebean leaves were kept in an observation room for normal cultivation at $24{ }^{\circ} \mathrm{C}-27{ }^{\circ} \mathrm{C}$. Mortality was assessed $48 \mathrm{~h}$ after treatment. Each test was run three times and results were averaged. Fenpyroximate was used as the control. Activities against $A$. medicaginis were evaluated by the similar procedure except that the culture temperature was reduced to $20^{\circ} \mathrm{C}-22{ }^{\circ} \mathrm{C}$. Inhibitions of $N$. lugens were tested on the rice seedlings, which was inoculated with $N$. lugens first. After that, the resultant rice seedlings were kept in an observation room for normal cultivation at $24^{\circ} \mathrm{C}-27^{\circ} \mathrm{C}$. Mortality was assessed $48 \mathrm{~h}$ after treatment. All of the tests were run with three duplicates and the results were averaged. Chlorantraniliprole and Abamectin were used as the positive controls, respectively.

\section{Conclusions}

In summary, a series of novel pyrazole oxime compounds containing isoxazole moiety were prepared and evaluated for their acaricidal activity against T. cinnabarinus, and insecticidal activities against $A$. medicaginis, $M$. separata and $N$. lugens. Bioassays results revealed that some title compounds exhibited potent acaricidal and insecticidal activities. Among these compounds, compound 9e showed $80.46 \%$ acaricidal activity against $T$. cinnabarinus at $500 \mu \mathrm{g} / \mathrm{mL}$, compounds $\mathbf{9 c}, \mathbf{9 h}, \mathbf{9 u}$, and $\mathbf{9 v}$ had $100.00 \%, 90.56 \%, 90.78 \%$, and $90.62 \%$ insecticidal activity against $A$. medicaginis at the concentration of $20 \mu \mathrm{g} / \mathrm{mL}$, respectively, compounds $9 \mathbf{k}$ and $9 \mathbf{u}$ possessed $70.86 \%$ and $100.00 \%$ insecticidal activity against $M$. separata at the dosage of $20 \mu \mathrm{g} / \mathrm{mL}$, respectively, and insecticidal activity against $N$. lugens of compounds $9 \mathbf{e}$ and $\mathbf{9 g}$ were $75.23 \%$ and $75.76 \%$ at $100 \mu \mathrm{g} / \mathrm{mL}$. Further analogue synthesis and structural optimization are well under way.

Supplementary Materials: The following are available online.

Acknowledgments: This work was funded by the National Natural Science Foundation of China (No. 21372135), the Research Foundation of the Six People Peak of Jiangsu Province (No. 2013-SWYY-013), the Technology Project Fund of Nantong City (Nos. CP12013002, MS22015020), and Postgraduate Research and Practice Innovation Program of Jiangsu Province (SJCX17_0640).

Author Contributions: Y.S. designed the research; H.D., W.Y., Y.F., S.S., J.C., J.S. performed the research and analyzed the data; G.J. wrote the paper. All authors read and approved the final manuscript.

Conflicts of Interest: The authors declare no conflict of interest.

\section{References}

1. Yu, M.; Liu, G.; Zhang, Y.; Feng, T.; Xu, M.; Xu, H. Design, synthesis and evaluation of novel isoxazolines/oxime sulfonates of 2(2,6)-(di)chloropodophyllotoxins as insecticidal agents. Sci. Rep. 2016, 6, 33062. [CrossRef] [PubMed]

2. Liu, G.; Ozoe, F.; Furuta, K.; Ozoe, Y. 4,5-Substituted 3-isoxazolols with insecticidal activity act as competitive antagonists of housefly GABA receptors. J. Agric. Food Chem. 2015, 63, 6304-6312. [CrossRef] [PubMed]

3. Xu, M.; Wagerle, T.; Long, J.K.; Lahm, G.P.; Barry, J.D.; Smith, R.M. Insecticidal quinoline and isoquinoline isoxazolines. Bioorg. Med. Chem. Lett. 2014, 24, 4026-4030. [CrossRef] [PubMed] 
4. Liu, Y.; Cui, Z.; Bin, L.; Cai, B.; Li, Y.; Wang, Q. Design, synthesis, and herbicidal activities of novel 2-cyanoacrylates containing isoxazole moieties. J. Agric. Food Chem. 2010, 58, 2685-2689. [CrossRef] [PubMed]

5. Hwang, I.T.; Kim, H.R.; Jeon, D.J.; Hong, K.S.; Song, J.H.; Cho, K.Y. 5-(2,6-Difluorobenzyl) oxymethyl-5-methyl-3-(3-methylthiophen-2-yl)-1,2-isoxazoline as a useful rice herbicide. J. Agric. Food Chem. 2005, 53, 8639-8643. [CrossRef] [PubMed]

6. Hamper, B.C.; Leschinsky, K.L.; Massey, S.S.; Bell, C.L.; Brannigan, L.H.; Prosch, S.D. Synthesis and herbicidal activity of 3-aryl-5-(haloalkyl)-4-isoxazolecarboxamides and their derivatives. J. Agric. Food Chem. 1995, 43, 219-228. [CrossRef]

7. Liu, P.; Xu, Y.; Li, J.; Liu, J.; Cao, Y.; Liu, X. Photodegradation of the isoxazolidine fungicide SYP-Z048 in aqueous solution: Kinetics and photoproducts. J. Agric. Food Chem. 2012, 60, 11657-11663. [CrossRef] [PubMed]

8. Piotrowska, D.G.; Andrei, G.; Schols, D.; Snoeck, R.; Grabkowska-Druzyc, M. New isoxazolidine-conjugates of quinazolinones-synthesis, antiviral and cytostatic activity. Molecules 2016, 21, 959. [CrossRef] [PubMed]

9. Kokosza, K.; Andrei, G.; Schols, D.; Snoeck, R.; Piotrowska, D.G. Design, antiviral and cytostatic properties of isoxazolidine-containing amonafide analogues. Bioorg. Med. Chem. 2015, 23, 3135-3146. [CrossRef] [PubMed]

10. Piotrowska, D.G.; Balzarini, J.; Glowacka, I.E. Design, synthesis, antiviral and cytostatic evaluation of novel isoxazolidine nucleotide analogues with a 1,2,3-triazole linker. Eur. J. Med. Chem. 2012, 47, 501-509. [CrossRef] [PubMed]

11. Kumar, R.N.; Dev, G.J.; Ravikumar, N.; Swaroop, D.K.; Debanjan, B.; Bharath, G.; Rao, A.G. Synthesis of novel triazole/isoxazole functionalized 7-(trifluoromethyl) pyrido[2,3- $d$ ]pyrimidine derivatives as promising anticancer and antibacterial agents. Bioorg. Med. Chem. Lett. 2016, 26, 2927-2930. [CrossRef] [PubMed]

12. Yu, G.J.; Iwamoto, S.; Robins, L.I.; Fettinger, J.C.; Sparks, T.C.; Lorsbach, B.A.; Kurth, M.J. 3-(Arylthiomethyl) isoxazole-4,5-dicarboxamides: Chemoselective nucleophilic chemistry and insecticidal activity. J. Agric. Food Chem. 2009, 57, 7422-7426. [CrossRef] [PubMed]

13. Sun, R.; Li, Y.; Xiong, L.; Liu, Y.; Wang, Q. Design, synthesis, and insecticidal evaluation of new benzoylureas containing isoxazoline and isoxazole group. J. Agric. Food Chem. 2011, 59, 4851-4859. [CrossRef] [PubMed]

14. Fu, C.; Pei, J.; Ning, Y.; Liu, M.; Shan, P.; Liu, J.; Zou, X. Synthesis and insecticidal activities of novel pyrazole oxime ether derivatives with different substituted pyridyl rings. Pest Manag. Sci. 2014, 70, 1207-1214. [CrossRef] [PubMed]

15. Song, H.; Liu, Y.; Xiong, L.; Li, Y.; Yang, N.; Wang, Q. Design, synthesis, and insecticidal evaluation of new pyrazole derivatives containing imine, oxime ether, oxime ester, and dihydroisoxazoline groups based on the inhibitor binding pocket of respiratory complex I. J. Agric. Food Chem. 2013, 61, 8730-8736. [CrossRef] [PubMed]

16. Yang, Y.; Lin, D.; Fu, C.; Zou, X. Synthesis and biological activity evaluation of novel pyrazole oxime ether derivatives containing chlorothiazole group and pyrimidine rings. Chin. J. Org. Chem. 2015, 35, 100-108. [CrossRef]

17. Li, Y.; Zhang, H.Q.; Liu, J.; Yang, X.P.; Liu, Z.J. Stereoselective synthesis and antifungal activities of (E)- $\alpha$-(methoxyimino) benzeneacetate derivatives containing 1,3,5-substituted pyrazole ring. J. Agric. Food Chem. 2006, 54, 3636-3640. [CrossRef] [PubMed]

18. Ouyang, G.; Cai, X.J.; Chen, Z.; Song, B.A.; Bhadury, P.S.; Yang, S.; Jin, L.H.; Xue, W.; Hu, D.Y.; Zeng, S. Synthesis and antiviral activities of pyrazole derivatives containing an oxime moiety. J. Agric. Food Chem. 2008, 56, 10160-10167. [CrossRef] [PubMed]

19. Park, H.J.; Lee, K.; Park, S.J.; Ahn, B.; Lee, J.C.; Cho, H.; Lee, K.I. Identification of antitumor activity of pyrazole oxime ethers. Bioorg. Med. Chem. Lett. 2005, 15, 3307-3312. [CrossRef] [PubMed]

20. Swanson, M.B.; Ivancic, W.A.; Saxena, A.M.; Allton, J.D.; O’Brien, G.K.; Suzuki, T.; Nokata, M. Direct photolysis of fenpyroximate in a buffered aqueous solution under a xenon lamp. J. Agric. Food Chem. 1995, 43, 513-518. [CrossRef]

21. Motoba, K.; Nishizawa, H.; Suzuki, T.; Hamaguchi, H.; Uchida, M.; Funayama, S. Species-specific detoxification metabolism of fenpyroximate, a potent acaricide. Pestic. Biochem. Physiol. 2000, 67, 73-84. [CrossRef] 
22. Dai, H.; Xiao, Y.S.; Li, Z.; Xu, X.Y.; Qian, X.H. The thiazoylmethoxy modification on pyrazole oximes: Synthesis and insecticidal biological evaluation beyond acaricidal activity. Chin. Chem. Lett. 2014, 25, 1014-1016. [CrossRef]

23. Dai, H.; Huang, J.; Jin, Z.; Cheng, X.; Huang, K.; Shi, Y.; Wang, Q.; Ling, Y. Synthesis and bioactivities of novel pyrazole oximes containing substituted thiadiazole moiety. Chin. J. Org. Chem. 2015, 35, 2617-2623. [CrossRef]

24. Wang, S.L.; Shi, Y.J.; He, H.B.; Li, Y.; Li, Y.; Dai, H. Synthesis and bioactivity of novel pyrazole oxime derivatives containing oxazole ring. Chin. Chem. Lett. 2015, 26, 672-674. [CrossRef]

25. Dai, H.; Li, G.; Chen, J.; Shi, Y.; Ge, S.; Fan, C.; He, H. Synthesis and biological activities of novel 1,3,4-thiadiazole-containing pyrazole oxime derivatives. Bioorg. Med. Chem. Lett. 2016, 26, 3818-3821. [CrossRef] [PubMed]

26. Park, M.S.; Park, H.J.; Park, K.H.; Lee, K.I. Introduction of N-containing heterocycles into pyrazole by nucleophilic aromatic substitution. Synth. Commun. 2004, 34, 1541-1550. [CrossRef]

27. Tanaka, A.; Terasawa, T.; Hagihara, H.; Sakuma, Y.; Ishibe, N.; Sawada, M.; Takasugi, H.; Tanaka, H. Inhibitors of acyl-CoA: Cholesterol O-acyltransferase. 2. Identification and structure-activity relationships of a novel series of $N$-alkyl- $N$-(heteroaryl-substituted benzyl)- $N^{\prime}$-arylureas. J. Med. Chem. 1998, 41, 2390-2410. [CrossRef] [PubMed]

28. Shi, Y.J.; Fang, Y.; Li, Y.; Chen, J.; Li, G.; Wang, Q.M.; Dai, H. Synthesis and biological activities of novel cyanoacrylate derivatives carrying 5-arylisoxazole group. Chem. J. Chin. Univ. 2017, 38, 421-428.

29. Yu, X.L.; Liu, Y.X.; Li, Y.Q.; Wang, Q.M. Design, synthesis, and acaricidal/insecticidal activities of oxazoline derivatives containing a sulfur ether moiety. J. Agric. Food Chem. 2015, 63, 9690-9695. [CrossRef] [PubMed]

30. Xu, R.B.; Xia, R.; Luo, M.; Xu, X.Y.; Cheng, J.G.; Shao, X.S.; Li, Z. Design, synthesis, crystal structures, and insecticidal activities of eight-membered azabridge neonicotinoid analogues. J. Agric. Food Chem. 2014, 62, 381-390. [CrossRef] [PubMed]

Sample Availability: Samples of the compounds $\mathbf{9 a - 9 v}$ and $\mathbf{1 3 a - 1 3 f}$ are available from the authors.

(C) 2017 by the authors. Licensee MDPI, Basel, Switzerland. This article is an open access article distributed under the terms and conditions of the Creative Commons Attribution (CC BY) license (http:/ / creativecommons.org/licenses/by/4.0/). 